\title{
Are C-rich ultra iron-poor stars also He-rich?
}

\author{
G. Meynet ${ }^{1}$, R. Hirschi ${ }^{2,3}$, S. Ekstrom ${ }^{1}$, A. Maeder ${ }^{1}$, C. Georgy ${ }^{1}$, P. Eggenberger ${ }^{1}$, and C. Chiappini ${ }^{1}$ \\ 1 Geneva Observatory, Geneva University, 1290 Sauverny, Switzerland \\ e-mail: georges .meynet@unige.ch \\ 2 Astrophysics Group, EPSAM Institute, University of Keele, Keele, ST5 5BG, UK \\ e-mail: r.hirschi@epsam.keele.ac.uk \\ 3 Institute for the Physics and Mathematics of the Universe, University of Tokyo, 5-1-5 Kashiwanoha, Kashiwa 277-8583, Japan \\ Received 30 September 2009 / Accepted 28 April 2010
}

\section{ABSTRACT}

\begin{abstract}
Context. The three most iron-poor stars presently known $([\mathrm{Fe} / \mathrm{H}]$ equal to $-5.96,-5.4$ and -4.75$)$ are carbon-rich, so they are called C-Rich Ultra-Metal Poor Stars (CRUMPS). The origin of their peculiar surface abundances is not understood.

Aims. We propose a synthetic view of the different models proposed so far to explain the peculiar abundances observed on the surface of the CRUMP stars. We deduce some expected trends based on nucleosynthetic arguments and look for signatures that allow models to be distinguished. We discuss the conditions for having CRUMP stars which are He-rich, i.e. with a mass fraction of helium greater than 0.30 and up to 0.60 .

Methods. We discuss the chemical composition of stars made of interstellar medium mixed with wind material of very metal-poor massive stars, with wind plus supernova ejecta and with material extracted from the envelope of early asymptotic giant branch (AGB) stars. Rotating and non-rotating models are considered.

Results. The high nitrogen abundances observed in CRUMP stars imply that the material that is responsible for their peculiar abundance pattern must be heavily enriched in primary nitrogen. We show that rotating stars (both massive and intermediate-mass stars) can produce the required amount of primary nitrogen, and can also account for the observed enhancements in $\mathrm{C}, \mathrm{O}, \mathrm{Na}, \mathrm{Mg}$, and Al. CRUMP stars formed from wind material of massive stars mixed with small amounts of pristine interstellar medium are He-rich (helium mass fraction between 0.30 and 0.60 ), Li-depleted, and present low ${ }^{12} \mathrm{C} /{ }^{13} \mathrm{C}$ ratios (inferior to 10 in number). Such He-rich stars, if discovered, would confirm that the most metal-poor CRUMPs formed from essentially pure wind/envelope material. They would provide the most direct way of probing the nucleosynthetic outputs of the first generations of stars.

Conclusions. We show that rotation is a key ingredient in explaining the abundance patterns of CRUMPS stars and probably also of at least some carbon-enhanced metal poor (CEMP) stars, in particuliar the CEMP-no stars. Similar non-rotating models, without any extra-mixing, do not succeed in explaining the enhancements in the three $\mathrm{CNO}$ elements.
\end{abstract}

Key words. stars: abundances - stars: rotation - stars: chemically peculiar - stars: AGB and post-AGB - stars: early-type nuclear reactions - nucleosynthesis, abundances

\section{Introduction}

The most iron-poor objects found to date in the Universe are halo field stars with $[\mathrm{Fe} / \mathrm{H}]$ as low as -5.96 (Frebel et al. 2008). Surprisingly, the three most iron-poor stars present strong overabundances of $\mathrm{C}$ with respect to Fe. More generally, Lucatello et al. (2006) find a lower limit of $21 \pm 2 \%$ for the number of stars with $[\mathrm{Fe} / \mathrm{H}] \leq-2.0$ and $[\mathrm{C} / \mathrm{Fe}] \geq+1.0$. These stars are collectively named C-Enhanced Metal-Poor (CEMP) stars. The C-Rich Ultra Metal Poor Stars (CRUMPS) are a subset of CEMP stars, those with $[\mathrm{Fe} / \mathrm{H}] \leq-4$. A more correct name would be C-rich ultra iron-poor stars. The overall metallicity, where the metals would contain not only iron but all the elements heavier than $\mathrm{He}$, would amount to an $[\mathrm{M} / \mathrm{H}]$ above -1 , which is, by far, not ultra-metal poor!

For some of these stars, strong overabundances of $\mathrm{N}, \mathrm{O}$, $\mathrm{Na}, \mathrm{Mg}$, and $\mathrm{Al}$ with respect to Fe have been found. Compared to solar values, the abundance ratios may be one to four orders of magnitudes greater depending on the element and/or star considered.

The CEMP stars can be classified into different categories according to the presence or absence of $s$ - and/or $r$-process elements (see the discussion in Masseron et al. 2010). In the present work, when comparisons are made with observations, we focus on the "CEMP-no" category, i.e., on those stars that present no evidence of enhancements in the $s$ - and $r$-process elements. The absence of $s$-process elements makes it less likely possible that the peculiar surface abundances of these stars result from accretion of material from an AGB companion, which is in general rich in $s$-process elements. As can be seen in Fig. 21 of Masseron et al. (2010), the "CEMP-no" category contains the most ironpoor objects and are thus the best candidates for studying the enrichment processes due to short-lived massive stars. Despite concentrating here on "CEMP-no" stars, the model studied in this work may also be relevant for at least some CEMP stars of other categories.

The main purpose is to discuss in detail the consequences of the "spinstar" model proposed by Meynet et al. (2006) and Hirschi (2007) where CRUMPS are formed from material ejected by metal-poor rotating stars (hereafter called the source material) mixed with some amount of interstellar material. We present the various outputs that can be obtained from such models and distinguish the following three possibilities for the origin of the source material:

1. the wind material ejected by one massive spinstar (spinstar wind model);

2. material ejected in the form of wind material during the whole massive spinstar lifetime, together with some amount 
of matter ejected at the time of the supernova event (spinstar wind and supernova model);

3. the envelope of an intermediate-mass spinstar at the early AGB phase (spinstar E-AGB model).

We discuss the main characteristics that may distinguish these various origins for the source material. In particular, we present the conditions required for producing CRUMP/CEMP-no stars, which in addition to being C-rich, are also He-rich.

The fact that some CEMP or CRUMP stars may be He-rich have important consequences for determining their physical characteristics:

- the mass determinations of the C-rich stars will be different depending on the abundance of helium considered. Typically, at any given position in the HR diagram, a star has a lower mass if it is He-rich;

- adopting an He-rich model atmosphere modifies the temperature and density structure of the outer layers where the absorption lines are formed. This may have an impact on the abundances deduced from spectral synthesis.

The discovery of very iron-poor He-rich stars in the field of the halo would be interpreted in the frame of the present model as the existence of stars made up of nearly "pure" source material. Such objects would therefore represent the most direct opportunities for studying the nucleosynthetic outputs of the first stellar generations.

Helium-rich stars are already indirectly found in some globular clusters (see e.g. Piotto et al. 2007). They may also be present in elliptical galaxies. All ellipticals show the UV upturn phenomenon, i.e. an UV excess, observed in their spectra. These excesses are caused by a population of stars on the blue end of the horizontal branch, i.e. by an old population of hot He-burning stars (see the review by O'Connell 1999). Different models have been proposed to explain the origin of this population (see e.g. Bressan et al. 1994; Han et al. 2007). A possibility, still to be explored, would be that these stars are the descendants of He-rich stars (Meynet et al. 2008a). Helium-rich low mass stars are indeed expected to populate the blue end of the horizontal branch (Decressin et al. 2009).

The paper is organized as follows. In Sect. 2, we recall the main characteristics of the "spinstar" model. Sections 3 to 5 discuss the chemical abundance of CRUMPS expected in the frame of the three possibilities listed above. In each case, we compare the theoretical expectations with the observed abundances in the most iron-poor stars known today and in CEMP-no stars. In Sect. 6 we briefly discuss consequences of alternative scenarios found in the literature. The isotopic ratio ${ }^{12} \mathrm{C} /{ }^{13} \mathrm{C}$ can be used to distinguish between different models. This is presented in Sect. 7. Conclusions and perspectives are given in Sect. 8.

\section{The "spinstar" model}

The term "spinstar" refers to those very metal-poor stars that rotate fast enough for their evolution to be strongly affected by rotation (Meynet et al. 2008b). Spinstars can be either massive or intermediate-mass stars.

Rotation is needed for two purposes. First, it allows mixing between the $\mathrm{He}$ - and the $\mathrm{H}$-burning regions and thus the synthesis of important amounts of primary ${ }^{13} \mathrm{C},{ }^{14} \mathrm{~N}$, and ${ }^{22} \mathrm{Ne}$. Rotation also deeply affects the $s$-process element nucleosynthesis (Pignatari et al. 2008). Second, rotation is important for triggering strong mass-loss episodes occurring during the evolution of these very metal poor stars. This is again due to rotational mixing, which transports primary elements to the surface. This increases the opacity of the outer layers and thus stimulates mass loss by line driven winds.

$A$ very metal poor environment $(Z$ below 0.001$)$ is needed because rotational mixing is more efficient at low metallicity (Maeder \& Meynet 2001). In addition, stars seem to rotate faster on average at low metallicities (see e.g. the review by Meynet et al. $2008 \mathrm{c}$, and references therein).

The effects discussed above are consequences of the same physics as, when applied to more familiar metallicities, are able to reproduce successfully many observed features, such as the changes in the surface abundances (Maeder et al. 2009), the correct number ratio of blue- to red-supergiants at low metallicity (Maeder \& Meynet 2001), the variation with metallicity in the number ratio of Wolf-Rayet (WR) to O-type stars, the existence of WR with surface abundances rich in both $\mathrm{H}$ - and He-burning products, the correct WN/WC number ratio at low metallicity (Meynet \& Maeder 2005), and the variation with metallicity in the ratio of type Ib and Ic to type II supernovae (Georgy et al. 2009).

With respect to the models reproducing the above observed trends, only two ingredients have been taken differently: obviously, the metallicity, which was chosen very low $\left(Z=10^{-8}\right.$ and $10^{-5}$ ), and the initial ZAMS rotational velocity on the equator. At such low metallicities, there is no guideline for choosing the initial rotational velocity, except that it should be inferior to the critical velocity (i.e., the velocity at which the centrifugal acceleration at the equator compensates for the local gravity). We chose ratios of $v_{\text {ini }} / v_{\text {crit }}$ between 50 and $70 \%$ on the ZAMS depending on the initial mass and metallicity considered (see Table 1). These values are well below 1 but above the usual ratio adopted for solar metallicity models, which is $40 \%$.

The general qualitative outline of the spinstar scenario is shown in Fig. 1. In A) a metal-poor rotating star evolves during the core H-burning phase. It may happen that the surface velocity reaches the critical velocity during this phase, forming an equatorial disk enriched in H-burning products. At the very low metallicity considered here, however, the amount of mass lost in that way is quite modest. B) During the core He-burning phase, as a result of rotational and convective mixing, which brings to the surface primary CNO elements, the opacity of the outer layers is increased. The star evolves to the red part of the HR diagram. A deep outer convective zone appears, dredging up large quantities of $\mathrm{CNO}$ elements to the surface. This triggers strong mass loss. The wind is made of both $\mathrm{H}$ - and He-burning products. C) Part of the wind material forms clumps. Some amount of interstellar medium may enter into their composition. If stars form later in these clumps, their initial composition will bear the mark of the composition of the spinstar's ejected material (source material). Sufficienly long-lived low-mass stars formed in that way produce the CRUMP stars that we observe today.

The end of the evolution of the spinstars, if massive, can be a black hole with no supernova explosion (D1). In that case there is no risk that the clumps be destroyed or further enriched by the supernova ejecta. The massive spinstar might also explode as a supernova (D2). In this case, the fast rotation of the core may produce a non-isotropic explosion (Tominaga et al. 2007a), which may allow some clumps to survive the explosion. More probably, an intermediate situation will arise where a faint supernova explosion occurs with a lot of material falling back on the nascent black hole as proposed by Umeda \& Nomoto (2003).

Enrichment of some clumps by supernova ejecta may occur. Therefore, in this general frame, various enrichment scenarios are possible depending on the nature of the spinstar, on the 
Table 1. Masses ejected by stellar winds from different rotating models and ${ }^{12} \mathrm{C} /{ }^{13} \mathrm{C}$ number ratios in the ejecta.

\begin{tabular}{clccccccccccc}
\hline \hline Model & $\begin{array}{l}M_{\text {ini }} \\
{\left[M_{\odot}\right]}\end{array}$ & $Z_{\text {ini }}$ & $\begin{array}{c}v_{\text {ini }} \\
{\left[\mathrm{km} \mathrm{s}^{-1}\right]}\end{array}$ & $v_{\text {ini }} / v_{\text {crit }}$ & $\begin{array}{c}M_{\text {eje }} \\
{\left[M_{\odot}\right]}\end{array}$ & $\begin{array}{c}M_{\mathrm{H}} \\
{\left[M_{\odot}\right]}\end{array}$ & $\begin{array}{c}M_{\mathrm{He}} \\
{\left[M_{\odot}\right]}\end{array}$ & $\begin{array}{c}M_{\mathrm{C} 12} \\
{\left[M_{\odot}\right]}\end{array}$ & $\begin{array}{c}M_{\mathrm{C} 13} \\
{\left[M_{\odot}\right]}\end{array}$ & $\begin{array}{c}M_{\mathrm{N} 14} \\
{\left[M_{\odot}\right]}\end{array}$ & $\begin{array}{c}M_{\mathrm{O} 16} \\
{\left[M_{\odot}\right]}\end{array}$ & ${ }^{12} \mathrm{C} /{ }^{13} \mathrm{C}$ \\
\hline $\mathrm{A}$ & 85 & $10^{-8}$ & 800 & 0.53 & 65.2 & 17.9 & 35.6 & 6.3 & 0.6 & 1.7 & 3.0 & 11.4 \\
$\mathrm{~B}$ & 60 & $10^{-8}$ & 800 & 0.57 & 11.0 & 7.2 & 3.8 & 0.000020 & 0.000005 & 0.000690 & 0.000050 & 4.3 \\
$\mathrm{C}$ & 60 & $10^{-8}$ & 800 & 0.58 & 36.2 & 14.5 & 21.5 & 0.005 & 0.001 & 0.2 & 0.006 & 5.4 \\
$\mathrm{D}$ & 40 & $10^{-8}$ & 700 & 0.55 & 4.2 & 2.9 & 1.3 & 0.005 & 0.0008 & 0.004 & 0.002 & 6.8 \\
& & & & & & & & & & & & \\
$\mathrm{E}$ & 60 & $10^{-5}$ & 800 & 0.72 & 23.1 & 10.9 & 12.2 & 0.0003 & 0.00007 & 0.010 & 0.003 & 4.6 \\
$\mathrm{~F}$ & 7 & $10^{-5}$ & 450 & 0.64 & 5.7 & 3.6 & 2.0 & 0.05 & 0.0007 & 0.02 & 0.004 & 77.4 \\
\hline
\end{tabular}

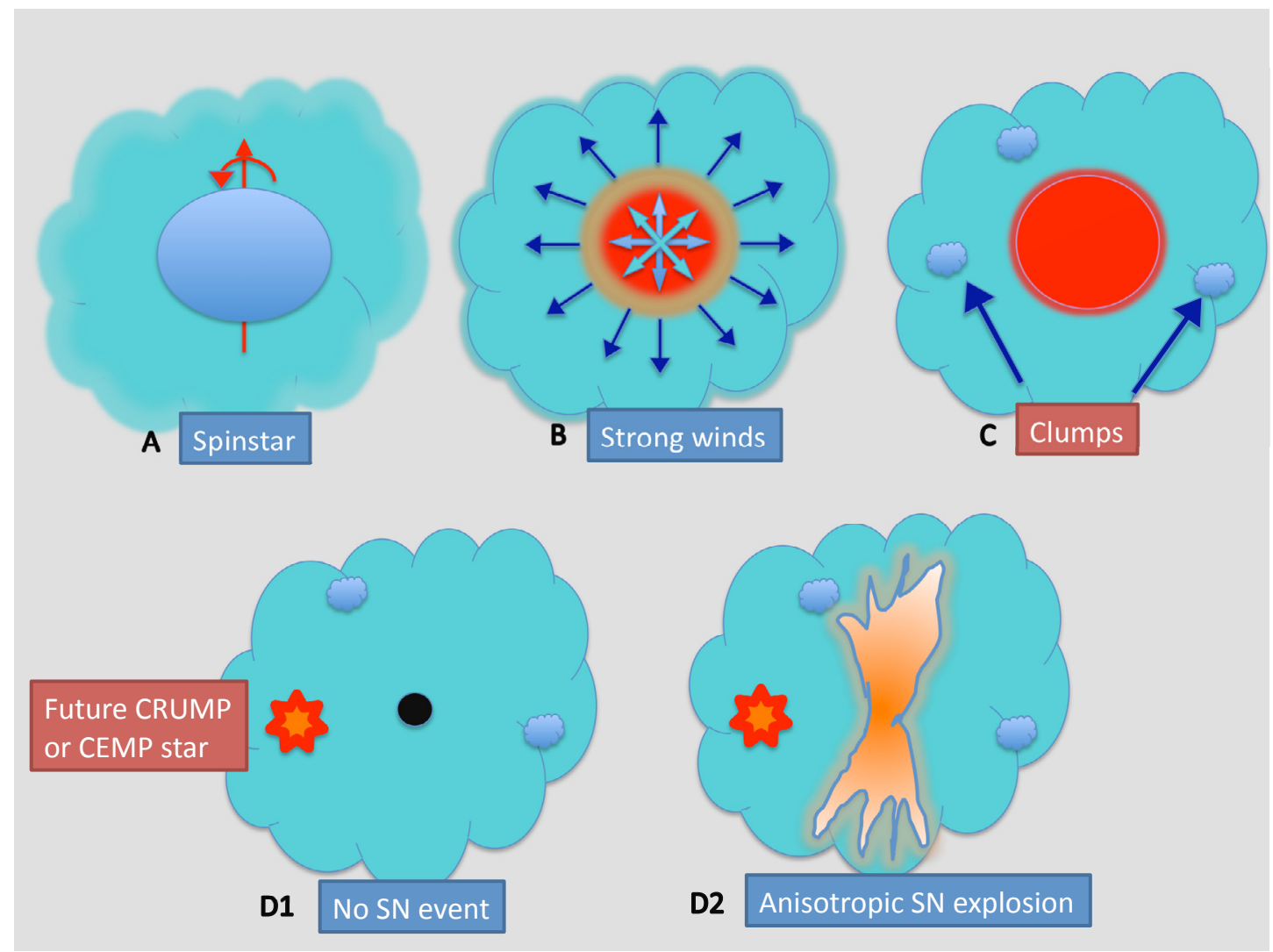

Fig. 1. Schematic representation of the "spinstar" model for explaining the origin of the peculiar abundance patterns of CRUMP/CEMP-no stars: A) A metal poor massive rotating star evolves during the core H-burning phase. B) During the core He-burning phase, strong winds, triggered by self enhancement of the surface metallicity appear. C) Wind material mixed with some amount of interstellar medium form clumps. The end of the evolution of the spinstars can either be a Black Hole with no supernova explosion (D1) or it can explode as a supernova (D2) which can further enrich the clumps. The fast rotation of the core may produce a non isotropic explosion. Some clumps form small mass stars, which are the CRUMP/CEMP stars that we can observe today.

degree of mixing with interstellar material, and on the importance of the wind's contribution with respect to the supernova one.

\section{The spinstar wind model}

In Fig. 2, we show how the surface abundances evolve as a function of the actual mass of the star (which decreases as a function of time) for a $60 M_{\odot}$ model at $Z=0.00001$ and with $v_{\text {ini }} / v_{\text {crit }}=0.72$. During the main sequence (MS) phase, the star loses only a little more than $6 M_{\odot}$. The material ejected during the MS phase bears the usual signature of the $\mathrm{CNO}$ cycle, i.e. increase in ${ }^{14} \mathrm{~N}$ and decrease in ${ }^{12} \mathrm{C},{ }^{16} \mathrm{O}$, and of the ${ }^{12} \mathrm{C} /{ }^{13} \mathrm{C}$ ratio. We note also the consequences at the surface of the action of the $\mathrm{Ne}-\mathrm{Na}$ and $\mathrm{Mg}-\mathrm{Al}$ chains, which are active in the $\mathrm{H}$-burning core (decrease in $\mathrm{Ne}$ and $\mathrm{Mg}$ isotopes and increase in ${ }^{23} \mathrm{Na}$ and ${ }^{27} \mathrm{Al}$ ). At any given point (in Fig. 2 when the actual mass is around $50 M_{\odot}$ ), He-burning products, such as ${ }^{12} \mathrm{C}$ and ${ }^{16} \mathrm{O}$ appear on the surface, while those due to $\mathrm{H}$ burning, such as nitrogen, are also increased. Rotational diffusion transports Heburning products in the H-burning shell where part of them are transformed by the $\mathrm{CNO}$ processing (explaining the strong ${ }^{13} \mathrm{C}$ 
A\&A 521, A30 (2010)

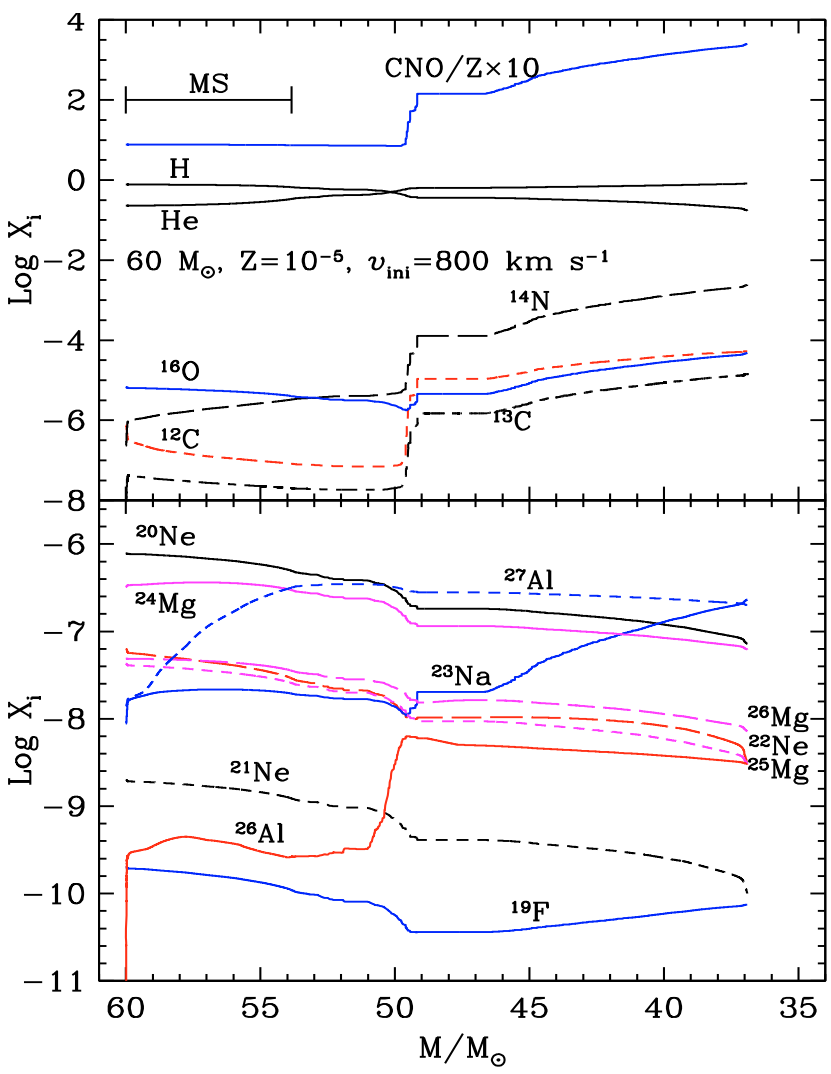

Fig. 2. Evolution of the surface abundances for a $60 M_{\odot}$ model with $Z=10^{-5}$ and $v_{\text {ini }}=800 \mathrm{~km} \mathrm{~s}^{-1}$ (model E in Table 1) as a function of the actual mass of the star. The actual mass decreases as a function of time. The extension of the main sequence (MS) phase is indicated in the upper panel. The curve labelled $\mathrm{CNO} / \mathrm{Z}_{\text {ini }}$ shows the evolution of the (logarithm of the) ratio $\left({ }^{12} \mathrm{C}+{ }^{14} \mathrm{~N}+{ }^{16} \mathrm{O}\right) / Z$ on the surface. The curve has been shifted to 1 dex upwards for clarity.

and ${ }^{14} \mathrm{~N}$ enhancements). Part of the He-burning products succeed in diffusing through the H-burning shell up to the surface without being destroyed by the $\mathrm{CNO}$ cycle (explaining the strong ${ }^{12} \mathrm{C}$ and ${ }^{16} \mathrm{O}$ abundances).

During the MS phase, the sum of the CNO element remains constant (see the upper curve in Fig. 2). When the actual mass of about $50 M_{\odot}$ is reached, the sum of CNO elements increases strongly as a result of rotational mixing in previous phases and the deepening of the outer convective zone. At that stage the star has an effective temperature of the order of 3.85 .

In Table 1, we indicate the following quantities for the various rotating models computed at very low metallicity: Cols. 2 to 5 give the initial mass, metallicity, the initial rotation velocity on the ZAMS, $v_{\text {ini }}$, and the ratio $v_{\text {ini }} / v_{\text {crit }}$ respectively. The total mass ejected by the winds is given in Col. 6. The total masses ejected in the form of $\mathrm{H}, \mathrm{He},{ }^{12} \mathrm{C},{ }^{13} \mathrm{C},{ }^{14} \mathrm{~N}$, and ${ }^{16} \mathrm{O}$ are given in Cols. 7 to 12 , respectively. The number ratio ${ }^{12} \mathrm{C} /{ }^{13} \mathrm{C}$ is indicated in Col. 13. The models for $Z=10^{-8}([\mathrm{Fe} / \mathrm{H}]=-6.6)$ are from Hirschi (2007) except for one, model C, taken from Meynet et al. (2006). Models at $10^{-5}([\mathrm{Fe} / \mathrm{H}]=-3.6)$ are from Meynet et al. (2006). Some differences in the input physics explain the different outputs for otherwise similar models (see the above references for more details).

From Table 1, it is easy to compute the initial chemical abundance that a star would have if formed from a mixture of wind material and of some amount of pristine interstellar matter. If $M_{\text {wind }}$ is the mass ejected in the form of wind and $M_{\mathrm{ISM}}$ the mass of interstellar material with which the wind material is mixed, then let us define $D$, the dilution factor, as the ratio $M_{\mathrm{ISM}} / M_{\text {wind }}$. Then the mass fraction of element $i, X_{i}$, in the star formed from wind ejecta and interstellar matter is given by

$X_{i}=\frac{\frac{M_{i}}{M_{\text {wind }}}+X_{i 0} \times D}{1+D}$,

where $M_{i}$ is the mass of element $i$ ejected by the winds, and $X_{i 0}$ the mass fraction of element $i$ present in the interstellar material. The quantity $\left[X_{i} / \mathrm{H}\right]$ can be computed from the formula

$$
\left[X_{i} / \mathrm{H}\right]=\log \left(\frac{\frac{M_{i}}{M_{\text {wind }}}+X_{i 0} \times D}{\frac{M_{\mathrm{H}}}{M_{\text {wind }}}+X_{\mathrm{H} 0} \times D}\right)-\log \left(\frac{X_{i \odot}}{X_{\mathrm{H} \odot}}\right),
$$

where $X_{i \odot}$ is the mass fraction of element $i$ in the $\operatorname{Sun}^{1}$. As interstellar abundances we used the initial abundances used to compute the models (Meynet et al. 2006; Hirschi 2007). The solar abundances are those of Asplund et al. (2005). These solar abundances are the same as those used for plotting the observed $[\mathrm{Xi} / \mathrm{H}]$ ratios in $\mathrm{HE} 1327-2326$ (see e.g. the values plotted in Fig. 4).

To compute the expected chemical composition of CRUMP stars made of such wind material, a first step consists in obtaining information on the dilution factor. This is the topic of the next section.

\subsection{Constraint on the dilution factor}

An interesting element to be used for constraining the dilution factor is Li. It was used by Decressin et al. (2007b) for estimating the dilution factor in another context, namely that of the chemical anomalies observed in globular clusters. We follow here the same line of reasoning in the context of the CRUMP stars found in the field of the halo.

Lithium has a reasonably well known value in the pristine interstellar medium equal to the value given by standard Big Bang nucleosynthesis. It is completely destroyed in massive stars and also in AGB stars. Thus any mixing of such stellar ejecta with pristine interstellar material will increase the abundance of $\mathrm{Li}$ with respect to the abundance in the source material. The dilution to be adopted is the one that will allow the observed $\mathrm{Li}$ abundance to be reproduced. Of course, this is correct as long as the abundance of $\mathrm{Li}$ is not affected by internal mixing processes having occurred in the CRUMP star itself.

For the star HE 1327-2326 $([\mathrm{Fe} / \mathrm{H}] \sim-5.96)$, Frebel et al. (2008) obtained an upper value of $\epsilon(\mathrm{Li})$ of 0.62 , with $\epsilon(\mathrm{Li}) \mathrm{de}-$ fined as $12+\log (N(\mathrm{Li}) / N(\mathrm{H}))$, where $N(\mathrm{Li})$ and $N(\mathrm{H})$ are the density number of $\mathrm{Li}$ and $\mathrm{H}$. This is a very low value, compared both to the abundance of Li expected from standard Big Bang nucleosynthesis (2.72 according to Cyburt et al. 2008), and to the value of Li observed in the bulk of normal metal-poor halo stars on the Spite plateau (2.10 according to Bonifacio et al. 2007).

As indicated above, HE 1327-2326 is either an evolved MS or an early subgiant star with an effective temperature of about $6200 \mathrm{~K}$. Although there are no "normal stars" (i.e. not C-enhanced) observed with such a low Fe content, hence no possibility of knowing the level of the Spite plateau in this range of Fe content, HE 1327-2326 presents all the characteristics (evolutionary stage and effective temperature) for belonging to the

\footnotetext{
1 For the quantities of the type [A/B], the result is the same using mass fraction or number fraction, provided of course the same type of quantities is used to compute the elemental abundances in the star and in the Sun.
} 
Spite plateau. Thus one would have expected a value around 2.10 instead of an upper limit of 0.62 .

Recently, Korn et al. (2009) have computed the effects of atomic diffusion including gravitational settling, thermal diffusion and radiative accelerations in models with masses around $0.78 M_{\odot}$ and an Fe content similar or lower than that of HE 13272326. In one model, an additional ad hoc diffusion coefficient is added with no attempt to connect it to a physical process like rotation. This diffusion coefficient was chosen to reproduce the observed abundance trends between stars from the turn off point to the red giant branch in NGC 6397 (Korn et al. 2007; Lind et al. 2008). For what concerns $\mathrm{Li}$, the model with no additional diffusion shows a depletion of $1.2 \mathrm{dex}$, and the one with additional diffusion shows a depletion by 0.3 dex. Thus we see that, according to this study, depletion mechanisms would not succeed in decreasing the $\mathrm{Li}$ abundance from the cosmological value below the observed upper limit. Indeed to obtain such a result, a depletion of at least 2.1 dex is required! Moreover, the case with no additional mixing, which produces the greatest depletion factor, is probably not the favored one since it cannot explain the observed trends in NGC 6397. From this we conclude that at the present time there is no strong reason to suppose that the very low Li abundance observed today in HE 1327-2326 results mainly from internal depletion processes. Thus we are left with the second possibility that the low Li-abundance observed today mainly results from the fact that this star was formed from very Li-poor material.

In the bottom panel of Fig. 3, the continuous lines show how the abundance of Li varies in a body formed from spinstar winds (completely Li-free) and of pristine interstellar material having WMAP Li abundance when the dilution factor is increased. The two lines corresponding to our 40 and $85 M_{\odot}$ models do not superpose because the wind ejecta of these two models do not have the same mean hydrogen abundance and $\epsilon(\mathrm{Li})$ show the variation in the abundance of $\mathrm{Li}$ with respect to $\mathrm{H}$ (see the definition just above).

Let us consider the case of the $40 M_{\odot}$ model. If no Li-depletion at all occurred in the CRUMP star, then the present day observed value is equal to the initial value. In that case, Fig. 3 tells us that the star should be made from pure ejecta. Any dilution would rapidly bring the $\mathrm{Li}$ abundance above the observed upper limit.

If the depletion factor is 1.2 dex (maximum and probably unrealistic value inferred from the models of Korn et al. 2009), then the observed upper limit would be compatible with a dilution factor inferior to about 0.13 (cf. the abscissa of the point on the $40 M_{\odot}$ model with an ordinate equal to $0.62+1.2=1.82$ ). Although not zero, the dilution factor remains extremely small.

We have also indicated in the bottom panel of Fig. 3 the upper limit abundance measured in 6 CEMP MS stars as obtained from the SAGA database of Suda et al. (2008). There are only 7 stars in total with measured $\mathrm{Li}$ abundance and low $[\mathrm{Fe} / \mathrm{H}]$. Except for one star, which is on the Spite plateau, the 6 other stars in this sample all have values of $\mathrm{Li}$ inferior to 1.75 , and the two stars in this sample with $[\mathrm{Fe} / \mathrm{H}]<-4$ have an upper limit for the $\mathrm{Li}$ abundance of 1.5 and 1.1, respectively. The upper limit of 1.75 is compatible with a dilution factor of 0.11 and 0.25 if there has been no Li-depletion or a 0.3 dex depletion in these CEMP stars. In case depletion of 0.6 dex is allowed (difference between the WMAP and Spite Plateau), then the dilution factor can be at most 0.65 .

From the discussion above we conclude that for a reasonable value of Li-depletion having occurred in the CRUMP star itself, then the low values of Li observed in some of the CEMP stars

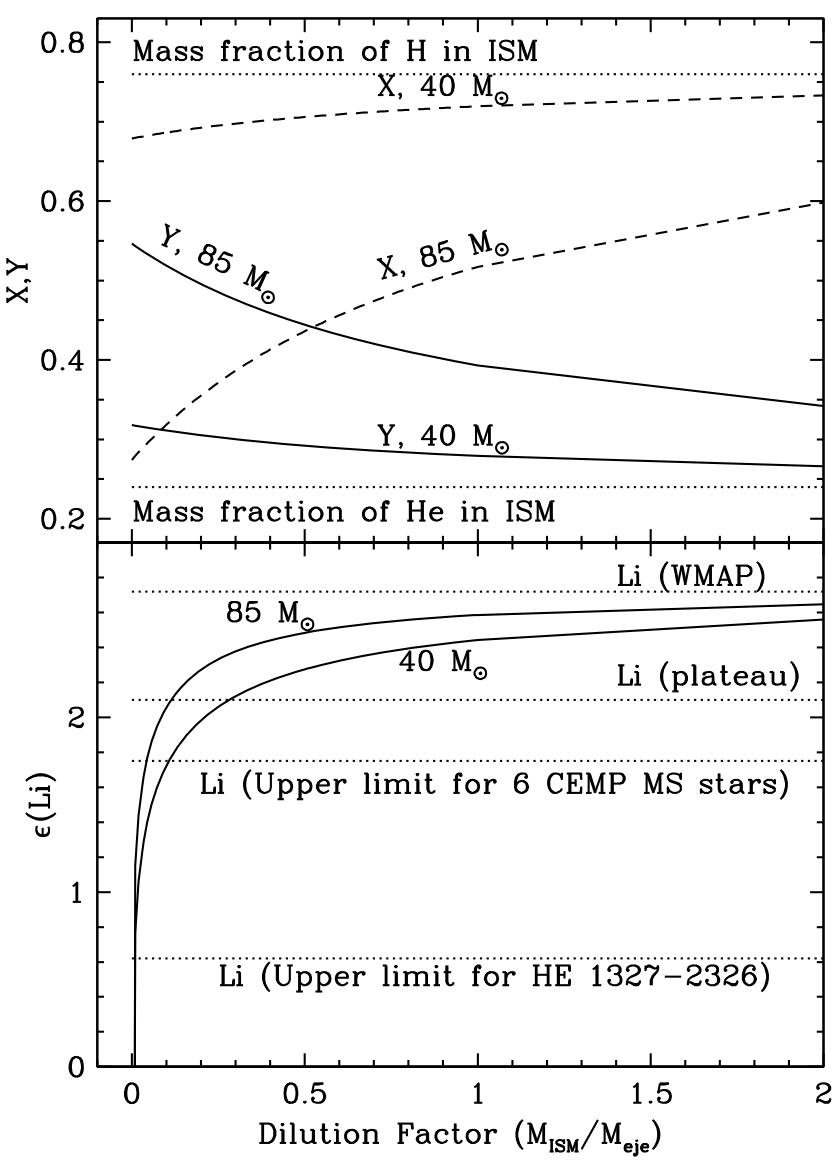

Fig. 3. Predicted abundances in stars made of a mixture of wind ejecta of massive rotating stars and of pristine interstellar medium plotted as a function of the dilution factor $D$, the ratio between the mass of interstellar medium and the mass ejected in the wind involved in the mixture. The rotating models are for 40 and $85 M_{\odot}$ models at $Z=10^{-8}$. The $85 M_{\odot}$ model has a rotational velocity on the ZAMS equal to $800 \mathrm{~km} \mathrm{~s}^{-1}$. The initial velocity for the $40 M_{\odot}$ model is $700 \mathrm{~km} \mathrm{~s}^{-1}$. In the upper panel, the mass fractions of hydrogen (dashed lines) and helium (continuous lines) are shown. The dotted lines indicate the mass fraction of these two elements in the interstellar medium. The lower panel shows the variation in the $\mathrm{Li}$ abundance as a function of the dilution factor for the two initial masses considered (continuous lines and see text for the meaning of $\epsilon(\mathrm{Li}))$. The WMAP cosmological abundance of $\mathrm{Li}$, the value of the $\mathrm{Li}$ abundance of the Spite plateau, the upper limit for the Li abundance observed in MS CEMP stars, and the upper limit observed in HE 1327-2326 (see references in the text) are indicated by horizontal dotted lines.

can only be explained if two conditions are fulfilled: 1) low LiCEMP stars are made of Li-free (or nearly free) material, and 2) a small amount of pristine interstellar medium can be added, at most $65 \%$.

Interestingly, this means that the appropriate abundances for the low Li-CEMP stars must be obtained mainly from the source. The dilution factor cannot be used as a free parameter allowing adjustment of the level of the abundances. This also implies that all the potential sources able to produce Li are discarded as candidates for providing low Li-CEMP stars material. 


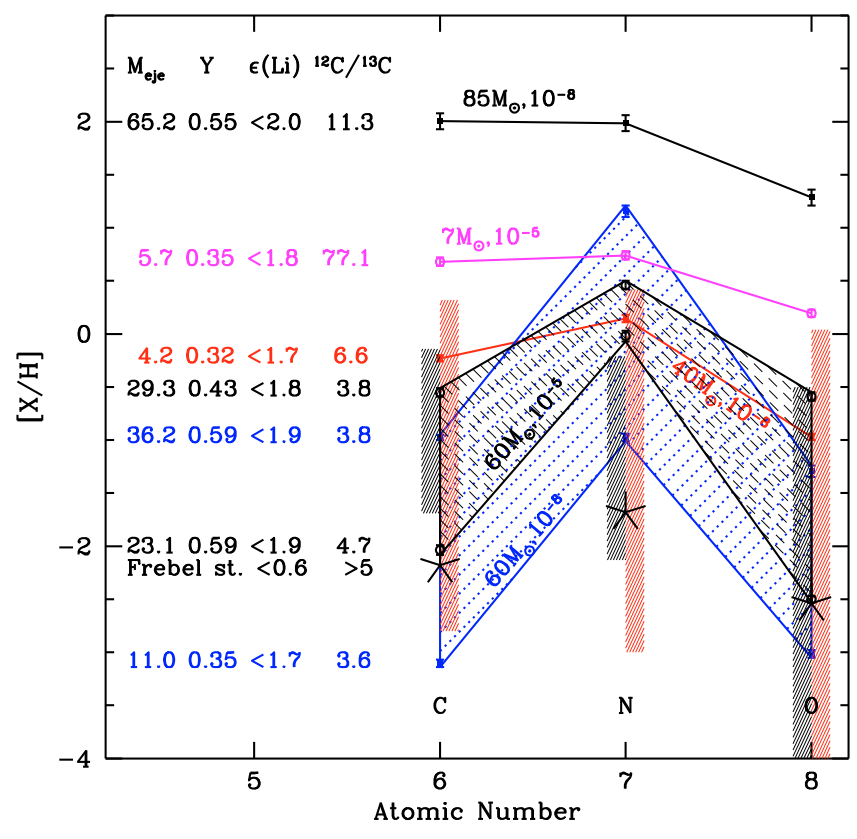

Fig. 4. The $[\mathrm{C} / \mathrm{H}],[\mathrm{N} / \mathrm{H}]$, and $[\mathrm{O} / \mathrm{H}]$ ratios in the wind ejecta of various rotating models mixed with at most $15 \%$ of pristine interstellar medium are shown (dilution factor $D=0.15$ ). The models are those presented in Table 1. The models obtained for the same initial mass, metallicity, and rotation, but computed with different prescriptions, cover the shaded areas. The vertical hatched columns show the range in observed values for CEMP-no stars with $\log g$ superior to 3.8 (left) and with $\log g$ inferior to 3.8 (right). The values were taken from Tables 1 and 2 of Masseron et al. (2010). Only upper limits are given for $[\mathrm{O} / \mathrm{H}]$, which is why the columns extend down to the bottom. The star symbols show the observed values for the most iron-poor star known today (Frebel et al. 2008). The left part of the figure indicates for each model the total mass ejected in solar masses, the mass fraction of $\mathrm{He}$ (case with $D=0$ ), the upper mass limit for $\mathrm{Li}$ abundance (obtained with $D=0.15$ ), and the isotopic ratio ${ }^{12} \mathrm{C} /{ }^{13} \mathrm{C}$ in number.

\subsection{Consequence of the small dilution factor: some CEMP stars are $\mathrm{He}$-rich}

In the upper panel of Fig. 3, we show how the abundance of He (in mass fraction) varies in a body formed from spinstar winds and of interstellar material with a mass fraction of $\mathrm{He}$ equal to 0.24 when the dilution factor is increased.

As can be seen from Table 1, the masses ejected by both the 40 and $85 M_{\odot}$ models are sufficient for forming at least one $0.8 M_{\odot}$ stars. Such a star, made of a pure rotating 85 (40) $M_{\odot}$ star wind ejecta would have an initial $Y \sim 0.55(0.32)$. For any dilution factors inferior to about $4.1(0.3)$, a CEMP star made of wind ejecta of a 85 (40) $M_{\odot}$ model, would have a He mass fraction superior to 0.30 .

This conclusion can be formulated in a more general way: any stars formed mainly from the envelope of an evolved massive or intermediate mass star, rotating or not, would be He-rich (see Table 2 in Meynet et al. 2010a). Thus He-richness would not be a hint per se that the spinstar model we propose here is correct but that the CRUMP stars are mainly formed from the envelope of evolved stars.

Would He-rich stars necessarily be Li-poor? The answer is no. The example above shows that even with a dilution factor of 4 (see the case of the $85 M_{\odot}$ model), we still have a Herich star, while the initial $\mathrm{Li}$ abundance, obtained with such a dilution factor, would be nearly equal to the cosmological value! Are Li-poor stars necessarily He-rich? No, because the CRUMP star may be formed from a high fraction of interstellar medium and Li may have then been depleted by internal mixing processes in the CRUMP star itself, although this is unlikely to happen in current stellar evolution models.

\subsection{Predictions for $C N O$ abundances in CRUMP/CEMP-no stars}

In Fig. 4, we show the values of the $[\mathrm{C} / \mathrm{H}],[\mathrm{N} / \mathrm{H}]$, and $[\mathrm{O} / \mathrm{H}]$ ratios obtained in the wind ejecta of our rotating models for dilution factors between 0 and 0.15 . Except for the $85 M_{\odot}$ (see error bars for the values of the $85 M_{\odot}$ model), the values obtained with $D=0$ and 0.15 are identical. The He mass fractions are indicated for a dilution factor equal to 0 . The upper value of the abundance of $\mathrm{Li}$ is given using $D=0.15$. The ratio ${ }^{12} \mathrm{C} /{ }^{13} \mathrm{C}$ is insensitive to such small variations in the dilution factor (see Sect. 7).

In Fig. 4, the vertical hatched zones show the range of observed values for CEMP-no stars with $\log g$ superior to 3.8 (left dark grey column, 5 stars in the sample) and with $\log g$ inferior to 3.8 (right light grey column, 20 stars in the sample, red in the online version). The observed data were taken from Table 2 of Masseron et al. (2010). The star symbols show the observed values for the most iron-poor star known today (HE 1327-2326, Frebel et al. 2008). The second (HE 0107-5240, Christlieb et al. 2004) and third (HE 0557-4840, Norris et al. 2007) most metalpoor star known today belong to the group of the CEMP-no stars with $\log g$ inferior to 3.8. These last two stars are giants, therefore their surface abundances may have been affected by the first dredge-up.

Before discussing the comparisons with the observed abundances in HE 1327-2326 and CEMP-no stars, we note a few general trends of the theoretical ratios:

- in the solar mixture, the CNO elements represent a number of atoms equivalent to about 80 times the number of $\mathrm{Fe}$ atoms, and the number of $\mathrm{N}$ atoms corresponds to about 3 times the number of Fe atoms. Let us suppose that these proportions are approximately the same at all metallicities. (This is the hypothesis we made for determining the initial distribution of the heavy elements in our model stars.) In that case, the maximum $[\mathrm{N} / \mathrm{H}]$ value one might expect if all the carbon and oxygen initially present in the star were transformed into nitrogen would be equal to $[\mathrm{N} / \mathrm{H}]_{\max }=$ $\log (80 / 3)+[\mathrm{Fe} / \mathrm{H}]=1.4+[\mathrm{Fe} / \mathrm{H}]$. Any $[\mathrm{N} / \mathrm{H}]$ value shifted upwards by more than about $1.4 \mathrm{dex}$ with respect to $[\mathrm{Fe} / \mathrm{H}]$ is an indication that nitrogen has to be produced in a primary process. This happens in all the computed models shown in Fig. 4;

- primary nitrogen can only be produced in a star that simultaneously has an $\mathrm{H}$ - and an He-burning region. In addition, some mixing process must occur between these two nuclear active regions. Rotational mixing is the mechanism we propose here to trigger this mixing. As recalled above, the mechanism that allows the ejection of that material is the self enrichment of the surface, also triggered by rotation. Thus rotational mixing, which produces the peculiar abundance pattern, also allows the chemically peculiar matter to be ejected by the star.

To see how these models compare with the observed abundances in HE 1327-2326, let us first note that this star presents strong signs of being made of material enriched by a very large quantity 
of primary nitrogen. Indeed the $[\mathrm{N} / \mathrm{H}]$ value of the order of -1.5 is well above the value of $-4.56=-5.96+1.4$ ! There are more than 3 dex of difference. All the present models show very strong productions of primary nitrogen with $[\mathrm{N} / \mathrm{H}]$ values shifted upwards by 2 to 4 dex with respect to $[\mathrm{Fe} / \mathrm{H}]$.

We see that the present models provide trends toward qualitative agreement with the observations of HE 1327-2326: low $\mathrm{Li}$ abundances and high $[\mathrm{X} / \mathrm{H}]$ ratios simultaneously for $\mathrm{C}, \mathrm{N}$, and $\mathrm{O}$, low ${ }^{12} \mathrm{C} /{ }^{13} \mathrm{C}$ (although only an upper limit is given for HE 1327-2326). All the models also predict He-rich $(Y \geq 0.30)$ stars. None of the present models, however, fits all the observed data perfectly. But here three facts need to be recalled. 1) The models are not at all adjusted to fit the observed abundances. We have simply plotted our predictions for the wind ejecta composition. As explained above, the dilution factor does not offer any degree of freedom. 2) Changes in the initial rotational velocities, in the initial masses, and probably, although to a lesser extent, in the initial metallicity would widen the range of values predicted by the models. 3) Depending on the evolutionary stage at which the mass is ejected by stellar winds, its chemical composition can vary a lot. This is the point we discuss in the next section.

Comparing the predictions of our spinstar wind models with the observations of CEMP-no stars (see hatched columns in Fig. 4), we see that the situation is generally rather encouraging. We see that many models cover the hatched regions.

\subsection{The spinstar shell model}

In this section we explore a slightly different version of the spinstar wind model explained in the previous section. In this model the CRUMP stars are still formed from spinstar ejecta but now, instead of considering the well-mixed material ejected by winds during the whole stellar lifetime, we consider shells of material ejected at different evolutionary stages. We still suppose that stellar winds are continuous but we consider that only wind material emitted during an interval of time is used to form the CRUMP star.

We begin by examining at which stage the wind ejected material presents the greatest similarities with the abundances observed on the surface of HE 1327-2326. In Fig. 5 the evolution of the $[\mathrm{X} / \mathrm{H}]$ ratios and of the number ratio ${ }^{12} \mathrm{C} /{ }^{13} \mathrm{C}$ are shown on the surface of the $60 M_{\odot}$ model with $Z=10^{-5}$ (model $\mathrm{E}$ in Table 1). The ${ }^{12} \mathrm{C} /{ }^{13} \mathrm{C}$ is very low most of the time (below 10 ), while the initial ratio used for computing the models is above 75 . However its evolution is not monotonic. It first decreases, as a result of the CNO cycle; then when He-burning products appear, a rapid increase in the ratio occurs (around $50 M_{\odot}$ in Fig. 5). Then owing to the continuous action of the H-burning shell it decreases again.

In Fig. 5 we have overplotted the level of the observed ratios in HE 1327-2326 as given by Frebel et al. (2008). The chemical composition of the wind has the greatest similarity with the abundances observed in the Frebel star when He-burning products begin to appear at the surface. Before that time, the matter would be depleted in $\mathrm{C}$ and $\mathrm{O}$, and after it, the enhancements of the $\mathrm{CNO}$ elements would be too high. Of course, the evolution shown in Fig. 5 changes when the initial mass of the star changes or when the initial velocity is varied.

In contrast to Fig. 5, where instantaneous values of the surface composition are plotted, the composition in shells of material is shown in Fig. 6. For instance, for our $85 M_{\odot}$ star, we consider 20 events of mass loss, each $3.25 M_{\odot}$ (summing a total of $65 M_{\odot}$ lost during the life of this star). Only the compositions of four or five shells are shown in each panel of Fig. 6. The

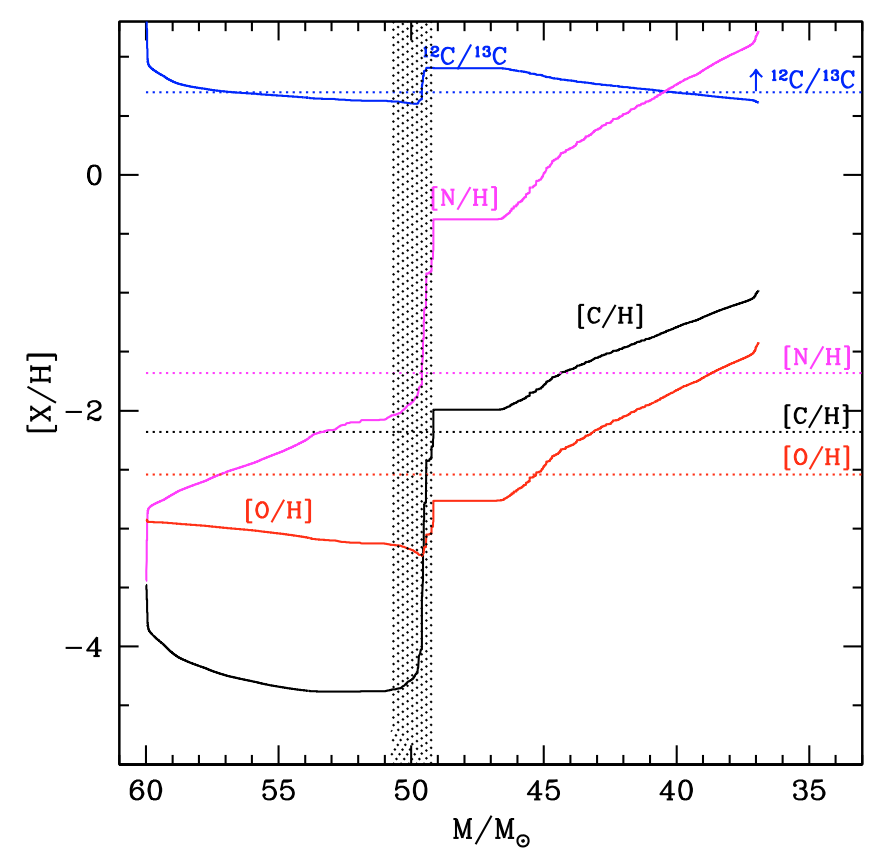

Fig. 5. Same as Fig. 2 for the $[\mathrm{X} / \mathrm{H}]$ ratios. The curve labelled ${ }^{12} \mathrm{C} /{ }^{13} \mathrm{C}$ shows the evolution in the number ratio of the two carbon isotopes. The horizontal dotted lines show the values obtained for HE 1327-2326 (Frebel et al. 2008). In the case of the carbon isotopic ratio, only a lower limit is given. The shaded area represents the portion of the mass ejected in the form of stellar winds which shows, once well mixed, the chemical composition plotted as the third curve from the bottom in left panel of Fig. 7.

lower curve corresponds to the first shell ejection and the upper one to the last one. When evolution proceeds, the $[\mathrm{X} / \mathrm{H}]$ 's shift upwards.

From Fig. 6 the following remarks can be made:

- already when the first shells are ejected, the ${ }^{12} \mathrm{C} /{ }^{13} \mathrm{C}$ ratio is quite low. It takes values near the one expected from $\mathrm{CNO}$ equilibrium. This occurs because this ratio is one of the first to change on the stellar surface. This is true for all the models discussed here;

- when evolution proceeds, some ${ }^{12} \mathrm{C}$ produced in the Heburning region does appear on the surface and increases the ${ }^{12} \mathrm{C} /{ }^{13} \mathrm{C}$ ratio to values above the equilibrium value. In further evolutionary stages, the continuous action of the $\mathrm{H}-$ burning shell again produces a decrease in this ratio. Thus, in the framework of the spinstar model, we see that $a$ value of the ${ }^{12} \mathrm{C} /{ }^{13} \mathrm{C}$ ratio slightly above the equilibrium $\mathrm{CNO}$ value, as observed in the Frebel star, favors material ejected at the transition between the phase when only H-burning products are ejected, and that when both $\mathrm{H}$ - and He-burning products are ejected. This confirms what was deduced for Fig. 5;

- as time goes on, matter processed by $\mathrm{H}$ - and He-burning are ejected and the CNO abundance ratios are enhanced by many orders of magnitude, as is the mass fraction of $\mathrm{He}$;

- in all three cases, there are shells that would fit the observed CNO abundances for the Frebel star (see e.g. the shell ejected at a mean age of 5.28 My by the $40 M_{\odot}$ stellar model). Similar cases might be found for the other models, changing the mass of the shells and/or the time interval during which it is ejected. 


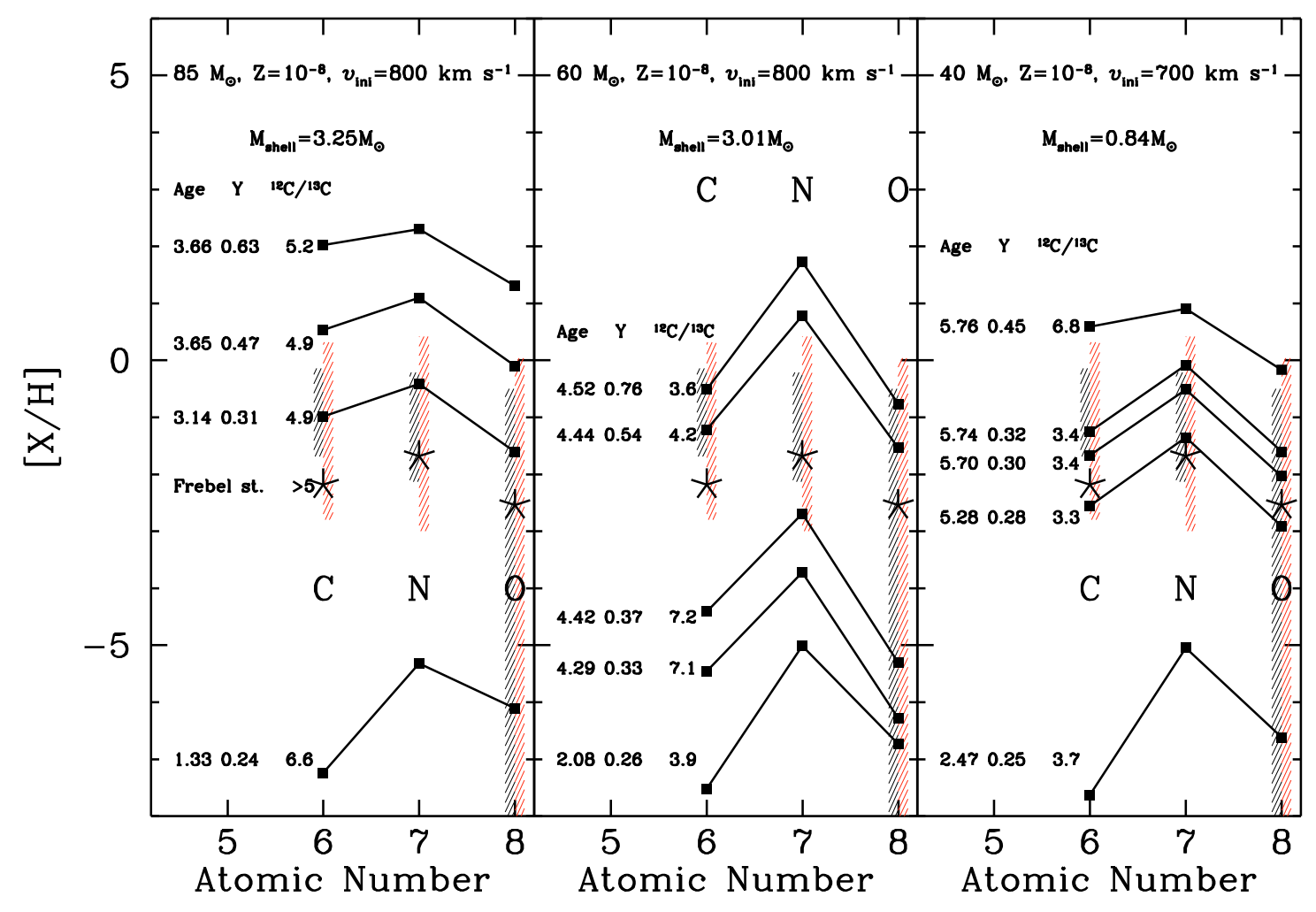

Fig. 6. The $[\mathrm{C} / \mathrm{H}],[\mathrm{N} / \mathrm{H}]$, and $[\mathrm{O} / \mathrm{H}]$ ratios in wind-shells ejecta of various rotating models are shown (see text). The models plotted are the models A, C, and D of Table 1. The masses of the shells are indicated along with the age of the star when half of the mass of the shell is ejected. The mass fraction of $\mathrm{He}$ and the ${ }^{12} \mathrm{C} /{ }^{13} \mathrm{C}$ in number in the shells are indicated (case with $D=0$ ). The stars show the observed values for the most iron-poor star known today (Frebel et al. 2008). The vertical hatched zones have the same meaning as in Fig. 4.

In Fig. 7 (left panel) we show similar results for the rotating $60 M_{\odot}$ at $Z=10^{-5}$ (model $\mathrm{E}$ in Table 1 ). For the CNO elements, we obtain qualitative similar results as at $Z=10^{-8}$. We can note that the best agreement with observation is also obtained for shells containing pure H-burning products and both $\mathrm{H}$ - and He-burning products.

\subsection{Predictions for $\mathrm{Na}, \mathrm{Mg}$ and $\mathrm{Al}$ abundances in CRUMP stars}

Since the $Z=10^{-5}$ models were computed accounting for the $\mathrm{Ne}-\mathrm{Na}$ and $\mathrm{Mg}-\mathrm{Al}$ chains, we show in the right panel of Fig. 7 the theoretical ratios expected for ${ }^{19} \mathrm{~F},{ }^{20,22} \mathrm{Ne},{ }^{23} \mathrm{Na},{ }^{24,25,26} \mathrm{Mg}$, and ${ }^{27} \mathrm{Al}$. The $\mathrm{Ne}-\mathrm{Na}$ and $\mathrm{Mg}-\mathrm{Al}$ chains are active in $\mathrm{H}$-burning regions. Their main effect is to transform $\mathrm{Ne}$ into $\mathrm{Na}$ and $\mathrm{Mg}$ into $\mathrm{Al}$. In the beginning, the shells have near solar $[\mathrm{X} / \mathrm{Fe}]$ ratios. (The curve corresponding to the first shell is the nearly horizontal line just above the $[X / F e]=0$ line, see right panel of Fig. 7.) The composition is not exactly solar because some surface enrichments have already occurred (see Fig. 2), and the initial composition adopted to compute the present models are slightly different from the Asplund et al. (2005) solar composition, which is used to normalise the plotted values.

Then when evolution proceeds, the general trend is the following: $[\mathrm{Ne} / \mathrm{Fe}]$ decreases and $[\mathrm{Na} / \mathrm{Fe}]$ increases, $[\mathrm{Mg} / \mathrm{Fe}] \mathrm{de}-$ creases and $[\mathrm{Al} / \mathrm{Fe}]$ increases, as is expected from the activity of the Ne-Na and Mg-Al cycle. The sawtooth shape of the abundance distribution pattern is more pronounced in shells ejected at the end of the evolution than at the beginning.
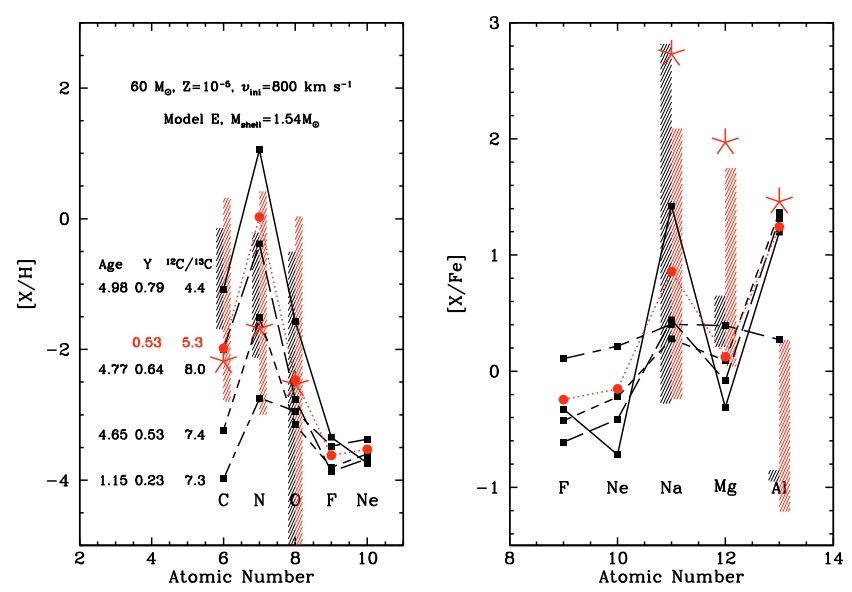

Fig. 7. Left panels: the $[\mathrm{C} / \mathrm{H}],[\mathrm{N} / \mathrm{H}],[\mathrm{O} / \mathrm{H}],[\mathrm{F} / \mathrm{H}]$ and $[\mathrm{Ne} / \mathrm{H}]$ ratios in different shells wind-ejected by models $\mathrm{E}$ of Table 1 . In the left part of the left panel are indicated for each shell the mean age at the time of shell ejection, the mean mass fraction of $\mathrm{He}$, and the isotopic ratio ${ }^{12} \mathrm{C} /{ }^{13} \mathrm{C}$ in number (case with $D=0$ ) in the shell. Right panels: the $[\mathrm{X} / \mathrm{Fe}]$ ratios are shown for ${ }^{19} \mathrm{~F},{ }^{20,22} \mathrm{Ne},{ }^{23} \mathrm{Na},{ }^{24,25,26} \mathrm{Mg}$, and ${ }^{27} \mathrm{Al}$. The stars show the observed values for the most iron poor star known today (Frebel et al. 2008). The hatched areas correspond to the range of values covered by observed values in CEMP-no stars. The left column corresponds to unevolved stars (sample of 6 stars), the right column to evolved stars (sample of 17 stars). The observations were taken from the SAGA database (Suda et al. 2008). 
Some effects might blur this simple picture, such as the arrival on the surface of some primary elements. During the core $\mathrm{H}$-burning phase, there is no possibility of producing primary $\mathrm{Na}$ and $\mathrm{Al}$, since there is no way for the star at this stage to produce $\mathrm{Ne}$ and $\mathrm{Mg}$. Thus $\mathrm{Na}$ and $\mathrm{Al}$ are built up from the initial $\mathrm{Ne}$ and $\mathrm{Mg}$ present in the star. During the core He-burning phase, some $\mathrm{Ne}$, through ${ }^{16} \mathrm{O}(\alpha, \gamma){ }^{20} \mathrm{Ne}$ in the most massive stars, and $\mathrm{Mg}$, through $\alpha$ captures on ${ }^{22} \mathrm{Ne}$, can be produced at the end of the core He-burning phase. Note that ${ }^{22} \mathrm{Ne}$ has a strong primary component in rotating models since this isotope is produced by the transformation of primary nitrogen, which has diffused from the H-burning shell into the He-burning core. Since the $\mathrm{Ne}$ and $\mathrm{Mg}$ are produced at the very end of the core $\mathrm{He}-$ burning phase, there is little time left for these elements to diffuse in the H-burning shell, to be transformed into $\mathrm{Na}$ and $\mathrm{Mg}$, and to be ejected into the stellar winds. In the present models, no primary components of $\mathrm{Ne}, \mathrm{Mg}, \mathrm{Na}$, and $\mathrm{Al}$ are present in the wind material.

Fluorine is built up in regions partially burnt by He-burning (periphery of the convective core during the core He-burning phase or of the He-burning shell). It results from transformation of part of the ${ }^{14} \mathrm{~N}$. Its abundance can be boosted by the production of primary nitrogen in rotating models. Since it is only produced in a relatively small part of the star, its apparition at the surface is very sensitive to many parameters of the models. In the model shown in Fig. 7, F is slightly depleted (with respect to $\mathrm{Fe}$ ) in the wind ejecta.

When we compare the global shape of the distribution pattern predicted in the shells with the observations, the first striking feature is the fact that the observations do not show the expected sawtooth pattern between $\mathrm{Mg}$ and $\mathrm{Al}$. Magnesium remains more abundant than $\mathrm{Al}$ in HE 1327-2326 and in all the CEMP-no stars for which the abundances of these two elements have been measured. Thus this seems to be a general feature and not just a peculiar circumstance.

In the frame of the sole activity of the $\mathrm{Mg}-\mathrm{Al}$ chain, such a pattern might be explained if the material had just been very partially processed by this chain. In that case $\mathrm{Mg}$ can be depleted very little while $\mathrm{Al}$ can be significantly increased. (Since initially $\mathrm{Mg} / \mathrm{Al}$ is about a factor 10 , the transformation of $10 \%$ of $\mathrm{Mg}$ into $\mathrm{Al}$ would already increase the $\mathrm{Al}$ abundance by a factor 2.) This may explain the relative abundance of $\mathrm{Mg}$ with respect of $\mathrm{Al}$. But one difficulty in that case would be to explain the absolute values. Since $\mathrm{Mg}$ would be depleted little, the high observed $[\mathrm{Mg} / \mathrm{Fe}]$ would correspond to nearly the initial $[\mathrm{Mg} / \mathrm{Fe}]$ ratio and this would indicate that at $[\mathrm{Fe} / \mathrm{H}]$ equal to -5.96 , the relative number of $\mathrm{Mg}$ atoms with respect to iron is about 100 times greater than the similar ratio in the Sun. Looking at the observations of Cayrel et al. (2004), this does not appear to be the case. In normal halo stars, values of $[\mathrm{Mg} / \mathrm{Fe}]$ around 0.2 are found down to an $[\mathrm{Fe} / \mathrm{H}]$ equal to -4.2 .

Another possibility would be that some $\mathrm{Mg}$ and $\mathrm{Al}$ produced in He-burning regions are ejected either in the winds or by the supernova. This is quite a reasonable solution, especially since a very tiny amount of core material would be sufficient. This can be seen in Fig. 10 where the chemical composition of material composed of the stellar winds plus supernova ejecta is shown for different values of the mass cut (see Sect. 4 for more details). We see that ejecting at the time of the supernova only the outermost $7 M_{\odot}$ stellar layers would allow for the correct $\mathrm{Mg} / \mathrm{Al}$ ratio (see curve with a mass cut $=30 M_{\odot}$ in Fig. 10). In that case, some dilution is needed to obtain the correct enrichment level. This is discussed in Sect. 4.

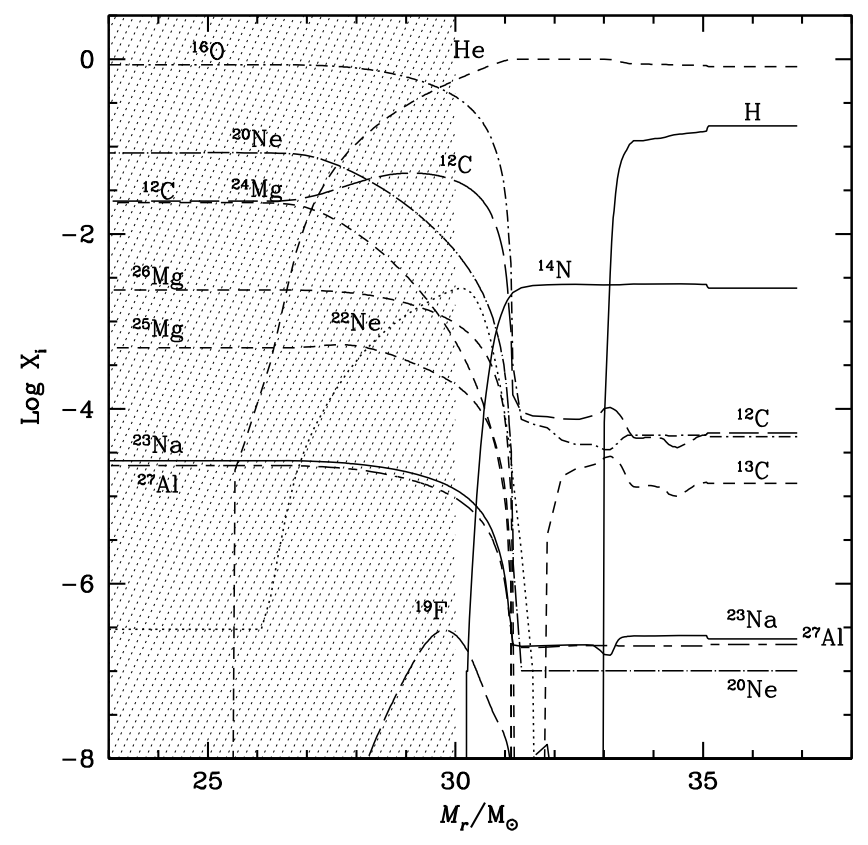

Fig. 8. Abundance profiles for various elements (in mass fraction) as a function of the Lagrangian mass in the outer layers of a $60 M_{\odot}$ model at $Z=10^{-5}$ with $v_{\text {ini }}=800 \mathrm{~km} \mathrm{~s}^{-1}$ at the end of the core He-burning phase (Model E in Table 1). The grey area represents the part of the star that would remain locked into the remnant when the mass cut is equal to $30 M_{\odot}$.

There is still one striking point to underline when looking at the right panels of Fig. 7: in most CEMP-no stars, $\mathrm{Al}$ is depleted with respect to Fe. This contrasts with the peculiar case of HE 1327-2326 and does appear difficult to reconcile with what we know about the nucleosynthesis of Al: no matter chain of reactions intervenes, $\mathrm{Al}$ is produced not destroyed! At that moment we only mention that $\mathrm{Al}$ abundances are determined from resonance lines that are very sensitive to NLTE effects (Cayrel et al. 2004). The abundances obtained from NLTE models might be 0.8 dex above the LTE abundances (Andrievsky et al. 2008)! In view of this, it might be premature to discuss solutions to that problem in too much details. We briefly come back to it in the next section.

\section{The spinstar wind and supernova model}

In Fig. 8, the chemical structure of our model E in Table 1 is shown. We make the hypothesis that the outer layers of this model already have, at the stage represented on this figure (end of core He-burning), the chemical composition they would have at the presupernova stage. This is only approximatively correct, but for the elements we are interested in here, it is a reasonable hypothesis.

For deciding what fraction of the mass is ejected at the time of the supernova explosion, one needs to know the mass cut between the ejected shells and the layers that will finally be incorporated into the compact stellar remnant. Here we consider the value of this mass cut as a free parameter. If this mass cut is chosen equal to the presupernova mass, then the supernova does not contribute to the enrichment of the ISM and the star will only contribute through its winds. When the mass cut is less than the presupernova mass, the mass ejected by the supernova is added to the mass ejected by the winds during the whole 
previous lifetime, and this constitutes the material composed of wind material and of supernova ejecta.

The composition of the wind+supernova material is shown in Fig. 10 for various values of the mass cut. Down to a mass cut of about $31 M_{\odot}$, the supernova ejecta would not change the composition a lot with respect to the composition of the wind material alone. As explained above, one sees the clear signature of the activity of the $\mathrm{Ne}-\mathrm{Na}$ and $\mathrm{Mg}$-Al chains. When He-burning regions are ejected, typically when the mass cut is decreased from 31 to $30 M_{\odot}$, a big change occurs. We see that $\mathrm{C}$ and $\mathrm{O}$ increase a lot, while the sawtooth pattern typical of the $\mathrm{Ne}-\mathrm{Na}$ and $\mathrm{Mg}$-Al chains is reversed. One notes also that the ${ }^{12} \mathrm{C} /{ }^{13} \mathrm{C}$ number ratio increases by orders of magnitude. A mass cut as low as $28 M_{\odot}$ and beyond would produce O-rich stars.

From Fig. 10, we can therefore deduce that to explain $\mathrm{N}$ abundances superior to those of $\mathrm{C}$ and $\mathrm{O}$ as observed in HE 1327-2326 for instance, the mass cut should be high, superior or equal to $30 M_{\odot}$ in the case of the model plotted in Fig. 10, and therefore the supernova contribution should be small. We see that adding deeper layers (lowering the mass cut below $30 M_{\odot}$ ) would rapidly lower the N/C and N/O ratios and thus prevent ratios higher than 1 as observed in HE 1327-2326. This means that most of the supernova ejecta fall back in the black hole and a little amount of the mass of the He-core is ejected (so not much C and $\mathrm{O}$ are finally ejected). This would be in line with the model proposed by Umeda \& Nomoto (2003). In that model, in addition to strong fall back, mixing is supposed to occur in a fraction of the He-core at the time of the explosion. This mixing process, which was found to take place in SN1987A and various explosion models (see the above reference), allows some iron, present in the deep layers, to be dredged-up in the ejected material. The mixing and fallback model also mimics the effect of asymmetric explosions in which matter from deep layers is ejected along the poles, while most of the matter falls back on the remnant at other lattitudes. Such models might be invoked for explaining possibly negative $[\mathrm{Al} / \mathrm{Fe}]$ ratios, while still maintaining $\mathrm{CNO}$ overabundances compatible with those observed in CRUMPS.

It is interesting to compare these results with those that would be obtained from a non-rotating model. The chemical structure of such a model is shown in Fig. $9^{2}$. The differences are striking. The most important ones are the following. The rotating model has lost more than $23 M_{\odot}$ by stellar winds, while the non-rotating model has lost nearly no mass. The envelope of the rotating model is enriched in primary ${ }^{12} \mathrm{C},{ }^{13} \mathrm{C},{ }^{14} \mathrm{~N}$ and ${ }^{16} \mathrm{O}$. The envelope of the non-rotating model presents the usual marks of CNO processing, without primary production of any of the $\mathrm{CNO}$ isotopes. The He-burning shell of the rotating model is significantly enriched in primary ${ }^{19} \mathrm{~F}$ and ${ }^{22} \mathrm{Ne}$, and the $\mathrm{CO}$ core has a mass of $30 M_{\odot}$. In the non-rotating model, there is no significant primary ${ }^{19} \mathrm{~F}$ and ${ }^{22} \mathrm{Ne}$, and the $\mathrm{CO}$ core has a mass of $18.2 M_{\odot}$.

The chemical composition of the material composed (mainly) of supernova ejecta from the non-rotating model is shown in Fig. 10 for two values of the mass cut. (The curves for the non-rotating models have been shifted downwards by 2.7 for clarity, see caption.) When the mass cut is chosen so that only CNO processed material is ejected (mass cut $=23 M_{\odot}$ ), then we obtain $\mathrm{N}$ enhanced with respect to $\mathrm{C}$ and $\mathrm{O}$. The absolute values of $\mathrm{C}$ and $\mathrm{O}$ are very low, of the order of -4 for $[\mathrm{C} / \mathrm{H}]$ and of -3.5 for $[\mathrm{O} / \mathrm{H}]$, much lower than the values observed in CRUMP stars. The abundance of $\mathrm{N}$ is also low, even accounting for the value

\footnotetext{
${ }^{2}$ Since that model was computed without accounting for the $\mathrm{Ne}-\mathrm{Na}$ and $\mathrm{Mg}-\mathrm{Al}$ chains, these elements are not shown in the figure.
}

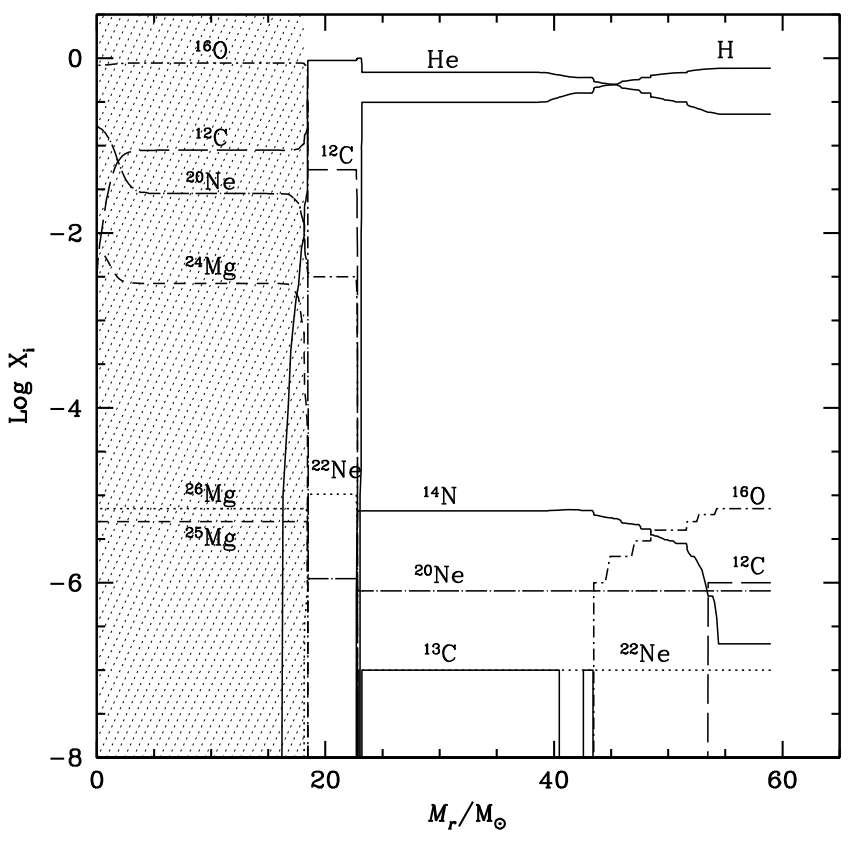

Fig. 9. Same as Fig. 8 for a non-rotating $60 M_{\odot}$ model at $Z=10^{-5}$. Models computed by Meynet $\&$ Maeder (2002). The grey area represents the part of the star that would remain locked into the remnant when the mass cut is equal to $18.2 M_{\odot}$.

plotted in Fig. 10 being shifted by 2.7 dex. The model plotted here has an initial metallicity equal to $[\mathrm{Fe} / \mathrm{H}] \sim-3.3$. Had we taken an $[\mathrm{Fe} / \mathrm{H}] \sim-6$, the abundance of nitrogen would be 2.7 dex lower (i.e. would be at the position plotted in Fig. 10). When the mass cut is $23 M_{\odot}$, the ${ }^{12} \mathrm{C} /{ }^{13} \mathrm{C}$ number ratio is quite low in the non-rotating model ejecta. It is typical of the CNO cy$\mathrm{cle}^{3}$.

When the mass cut is chosen to be equal to $18.2 M_{\odot}, \mathrm{N}$ is not significantly changed, while $\mathrm{C}$ and $\mathrm{O}$ are increased by many orders of magnitudes. The ${ }^{12} \mathrm{C} /{ }^{13} \mathrm{C}$ number ratio also reaches much higher ratios than in the material ejected by the rotating model. In Fig. 11, we show the composition obtained by the wind and supernova models accounting for a dilution factor of about 100 with pristine interstellar medium. We obtain a reasonable agreement using our rotating model. In that case, the large dilution factor means the star would not be He-rich. Its ${ }^{12} \mathrm{C} /{ }^{13} \mathrm{C}$ ratio would reach a high value of around 190. Thus such a model would have difficulty explaining unevolved CEMP stars showing low ${ }^{12} \mathrm{C} /{ }^{13} \mathrm{C}$ ratios. Such a model would also require an efficient depletion process for $\mathrm{Li}$.

The non-rotating model would predict C-rich stars, but without strong enhancement of nitrogen (only secondary nitrogen). Such a model would also predict an extremely high value for the ${ }^{12} \mathrm{C} /{ }^{13} \mathrm{C}$ ratio. With dilution, $D=100$, the ratio would be about 1550 .

\section{The spinstar AGB model}

As explained in Sect. 6.3, AGB stellar models are invoked as a possible cause for the CEMP stars. In the models published

\footnotetext{
${ }^{3}$ However, since here $\mathrm{C}$ and $\mathrm{O}$ abundances depend on the initial abundances, any mixing with interstellar material would rapidly increase the ${ }^{12} \mathrm{C} /{ }^{13} \mathrm{C}$ number ratio up to its value in the interstellar medium.
} 


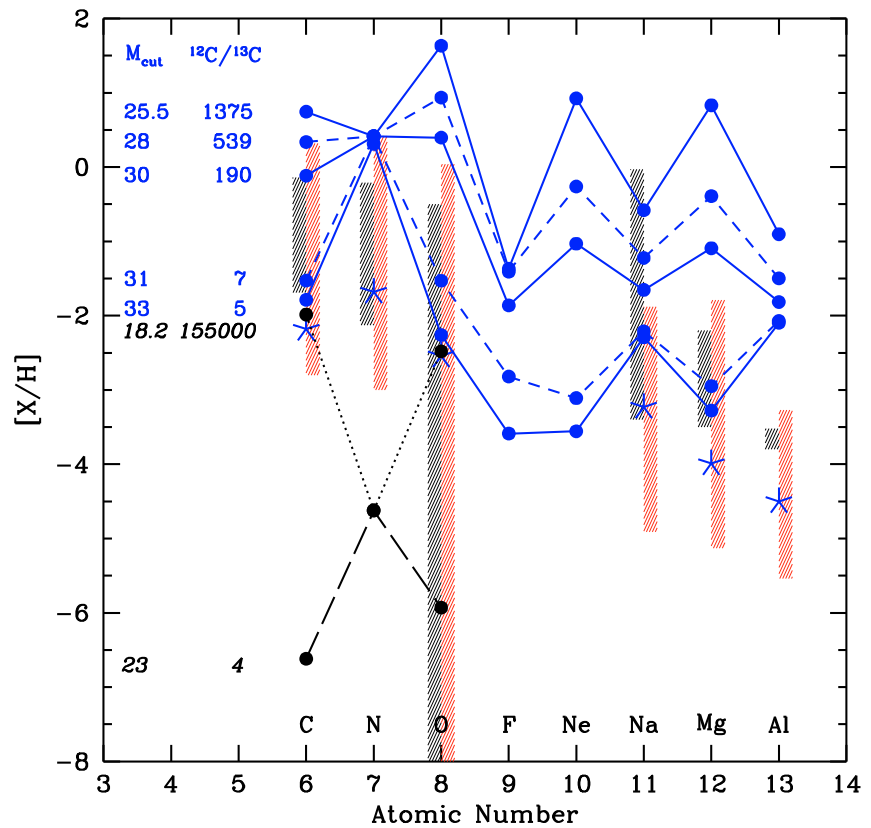

Fig. 10. Composition of the mixture composed of wind material of $60 M_{\odot}$ models at $Z=10^{-5}$ and of layers ejected by the supernova. The upper curves (continuous and short dashed lines) correspond to the rotating model with $v_{\text {ini }}=800 \mathrm{~km} \mathrm{~s}^{-1}$ (model E in Table 1). The lower curves (dotted lines and long dashed curves) correspond to the nonrotating models (models from Meynet \& Maeder 2002). In those last cases, the curves were shifted downwards by 2.7 dex for clarity. Various values of the mass-cut are considered. The ${ }^{12} \mathrm{C} /{ }^{13} \mathrm{C}$ number ratios in the mixture are also indicated for each case.

until now (see e.g. Suda et al. 2004), the CEMP star has acquired its characteristic surface composition because it accreted some AGB wind material at its surface. The model we present here is different in at least two respects. First we suppose that the AGB star that provides the peculiar chemical composition material originates in a star that was a fast rotator during the MS phase. Second, we suppose that the whole CEMP star, or at least a large part of it, is made from a mixture of AGB envelope and of pristine interstellar medium, not just its $0.01 M_{\odot}$ exterior layers as assumed by Suda et al. (2004). The situation in the present model is similar to the one sketched in Fig. 1, except that, in panels A to $\mathrm{C}$, instead of having a massive star, we consider here the case of an intermediate mass star (panels D1 and D2 of course do not apply in that case.)

Before making the comparisons with observed abundances at the surface of CEMP-no stars, we want to make two remarks. First, our $7 M_{\odot}$ stellar mass model is in the early-AGB phase. During the subsequent phase, the thermal-pulse AGB phase, the chemical structure of the envelope will change and strong mass loss will occur. Thus the present models should be pursued in the TP-AGB phase before any more quantitative conclusions can be reached. At the moment, the results presented here may, however, be relevant in the frame of the close binary scenario. If our intermediate-mass star belongs to a close binary system, some mass transfer may occur when the primary inflates to join the early AGB. In that case some mass can be accreted by the secondary and/or lost by the system as a whole ${ }^{4}$.

\footnotetext{
${ }^{4}$ Such a scenario can occur in a binary wide enough for the star to evolve into the E-AGB phase, and tight enough so that mass transfer occurs during the E-AGB phase.
}

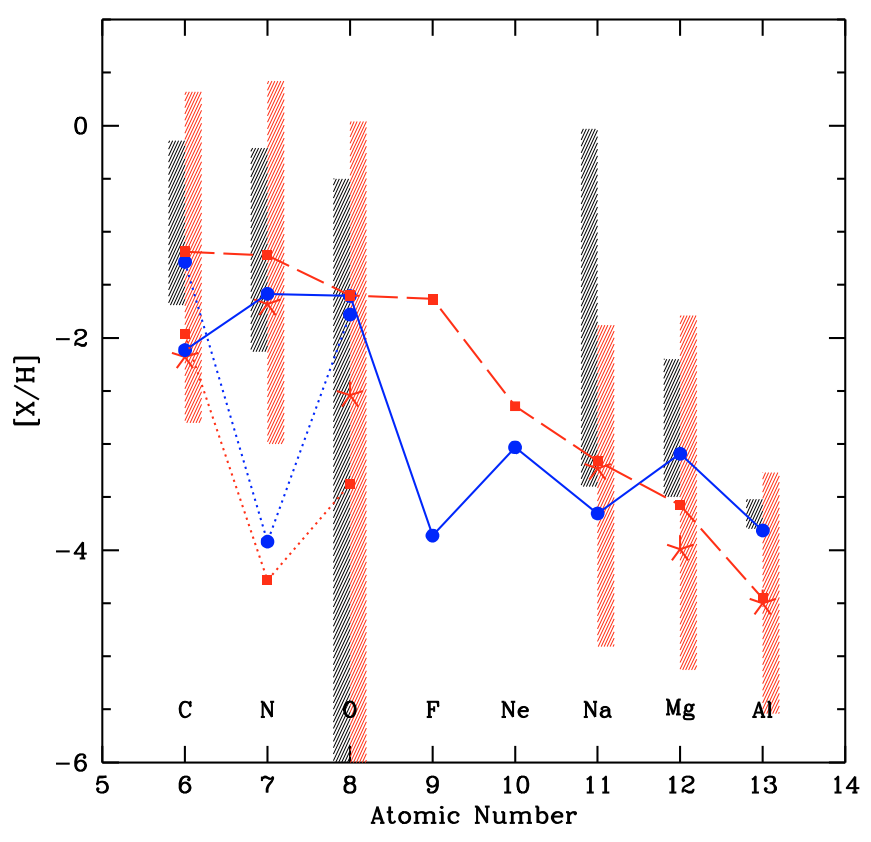

Fig. 11. Comparisons between abundances in various stellar model ejecta and the abundances observed in CEMP-no stars. The continuous curve with filled circles shows the composition of the mixture composed of material ejected by the rotating $60 M_{\odot}$ models at $Z=10^{-5}$ (wind and supernova, model $\mathrm{E}$ in Table 1) and of interstellar material. A dilution factor of 100 has been used. (Here the dilution factor is the mass of interstellar medium divided by the mass ejected by the $60 M_{\odot}$.) A mass cut equal to $30 M_{\odot}$ was chosen. The dotted curve with filled circles corresponds to the non-rotating $60 M_{\odot}$ stellar model with a mass cut of $18.2 M_{\odot}$. The same dilution factor was applied. The dashed line with squares shows the composition obtained by mixing the outer envelope (mass above the $\mathrm{CO}$ core) at the E-AGB phase of our rotating $7 M_{\odot}$ stellar model with interstellar medium (dilution factor equal to 100). The dotted curve with squares shows the composition obtained in the same manner (mass above the $\mathrm{CO}$ core and interstellar medium) using our non-rotating $7 M_{\odot}$ stellar model.

The second remark is that, according to the $Z=10^{-5}$ models of Meynet \& Maeder (2002), computed for initial velocities of $300 \mathrm{~km} \mathrm{~s}^{-1}$, similar behaviours are obtained at least down to $2 M_{\odot}$. Thus, rotational mixing will have an impact not only on the chemical structure of the most massive AGB stars, or those rotating fast, but also on the whole range of initial masses and rotational velocities.

In Figs. 12 and 13 the chemical structure of our rotating and non-rotating $7 M_{\odot}$ models at $Z=10^{-5}$ are shown. As for the most massive stars, the differences are striking and are qualitatively the same. The only point that is very different than the massive star case, is that, up to this point, even the rotating intermediate mass star model has lost very little from stellar winds, because the surface enrichments in the rotating model occurred just before the evolutionary stage shown in Fig. 12 and thus the enhanced mass loss had no time to peel off the star.

In the rotating model, in the zone between the He-burning shell (at about $1.3 M_{\odot}$ ) and the H-burning one (at $1.6 M_{\odot}$ ), the levels of primary ${ }^{13} \mathrm{C},{ }^{14} \mathrm{~N}$, and ${ }^{22} \mathrm{Ne}$ are higher than in the rotating $60 M_{\odot}$ model, because the $7 M_{\odot}$ evolves rapidly to the red part of the HR diagram at the end of the MS phase, as a result of the rapid core contraction and envelope expansion. Thus a strong $\Omega$-gradient is present at the border of the He-burning core during most of the He-burning phase, making an efficient diffusion of 


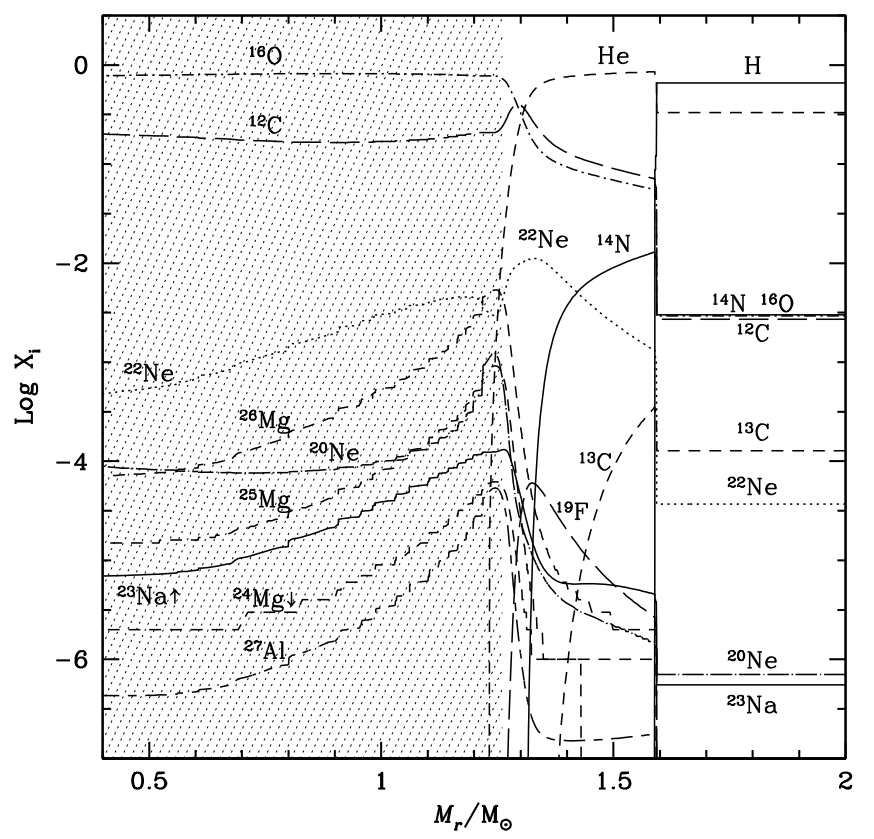

Fig. 12. Variation in the abundances of various elements (in mass fraction) as a function of the Lagrangian mass in the outer layers of a $7 M_{\odot}$ model at $Z=10^{-5}$ with $v_{\text {ini }}=450 \mathrm{~km} \mathrm{~s}^{-1}$ at the end of the core Heburning phase (model $\mathrm{F}$ in Table 1). The grey area corresponds to the CO-core.

He-burning products into the H-burning shell and of H-burning products into the He-burning core possible.

In Fig. 11 we show the composition of a star made of the envelopes of such AGB stars mixed with interstellar material (dilution factor equal to 100). We see that we obtain a reasonable fit if we consider our rotating model. Such a star would not be $\mathrm{He}$-rich, and would present a ${ }^{12} \mathrm{C} /{ }^{13} \mathrm{C}$ ratio equal to 104 . Our non-rotating model cannot account for the high nitrogen abundance. Moreover, the ${ }^{12} \mathrm{C} /{ }^{13} \mathrm{C}$ ratio would be close to 8000 (after dilution).

The spinstar AGB model would, however, have some difficulty explaining the lower-than-solar $[\mathrm{Al} / \mathrm{Fe}]$ ratios (if real!). Such stars do not eject any Fe, while they inject some Al, thus they would predict positive $[\mathrm{Al} / \mathrm{Fe}]$ values as is observed in HE 1327-2326!

\section{Alternative models}

In this section we briefly discuss alternative models for explaining CRUMPS. They invoke either AGB stars in binary systems or supernovae. Venn \& Lambert (2008), on the other hand, suggest that the abundance pattern observed today on the surface of CRUMPS does not reflect the initial abundances of the cloud from which the star was born, but results from processes occurring in or around the star. Let us begin by examining this hypothesis.

\subsection{Are CRUMPS produced by in situ processes?}

Venn \& Lambert (2008) suggest that CRUMPS are not actually all that metal poor, but may have their photosphere deprived in heavy elements owing to a separation of gas and dust beyond the stellar surface, followed by the accretion of dust-depleted gas. Such a process is invoked to explain Lambda Bootis stars,

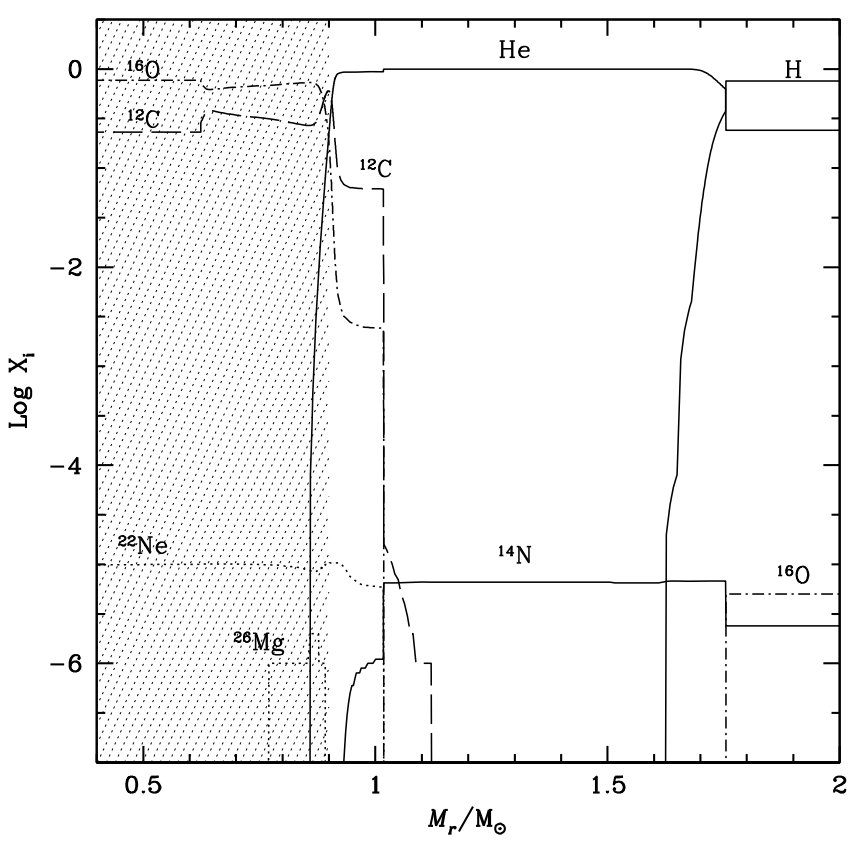

Fig. 13. Same as Fig. 12 for a non-rotating $7 M_{\odot}$ model at $Z=10^{-5}$, as computed by Meynet \& Maeder (2002). The grey area corresponds to the CO-core.

which are MS stars with a spectral type from A to mid-F, found all along the MS phase. They are expected to have a solar-like composition but show significant metal deficiencies. The authors propose some tests of their hypothesis, for instance looking at the abundances of sulphur and zinc. Dust formation does not deplete these two elements much so that in the framework of this scenario, they should not show the same depletion as the rest of metals. Unfortunately, no such observations are available yet.

While this explanation is indeed quite interesting, it can probably not be invoked to explain all the observed cases. Our main argument is the following: if the dust-gas separation process may produce different elemental ratios, it should not change isotopic ratios! Such a scenario has difficulty explaining why, for instance in G77-6 (Plez \& Cohen 2005), which is an unevolved star, values as low as 5 of the ${ }^{12} \mathrm{C} /{ }^{13} \mathrm{C}$ ratio have been measured. This value is well below the ${ }^{12} \mathrm{C} /{ }^{13} \mathrm{C}$ ratios measured on the surface of stars belonging to the "unmixed" sample of red-giants metal-poor stars observed by Spite et al. (2006), so results from a nuclear process that occurred in stars of previous generations and not from an in-situ fractionation process.

\subsection{Non-rotating supernovae models}

CRUMPS might form from material made of a mixture of supernova ejecta (originating in non-rotating progenitors) and of interstellar material. One question that immediately arises when suggesting this origin for CRUMPS is why they are produced at very low metallicity. From physical considerations, one expects that supernovae developing from zero metallicity or very metal poor progenitors present different characteristics from supernovae coming from solar metallicity progenitors. At zero or very low metallicity, progenitors are more compact. This favors a higher amount of fallback (Chevalier 1989). The more compact structure of the presupernova at very low metallicity reduces 
the extent of the region where Rayleigh-Taylor instabilities grow and where the mixing of the ejecta can occur. A consequence of this is that the yields of such a supernova explosion are very sensitive to the amount of fallback. Thus it may be that the ejecta at very low metallicity present very different characteristics from those expected at standard metallicities.

Many models invoking supernovae have been proposed to explain the peculiar abundance pattern of CEMP stars (Umeda \& Nomoto 2003; Limongi et al. 2003; Tominaga et al. 2007b; Joggerst et al. 2009). For instance, the zero-metallicity models of Joggerst et al. (2009) show overproductions of $\mathrm{C}$ and $\mathrm{O}$ compared to $\mathrm{Fe}$, a trend corresponding to what is observed in CRUMPS. However, such models have difficulty reproducing the strong $\mathrm{N}$ overabundances, quite in line with what we obtained with our non-rotating models (see above). As indicated by these authors, accounting for the effects of rotation might help in resolving this difficulty.

Another difficulty that may emerge from these models is that they need a very high dilution factor with a pristine interstellar medium to reproduce the observed levels of the overabundances. As we have seen this is contrary to the observed very low Li abundances unless depletion mechanisms that are much stronger than those usually accounted for in current models are supposed $^{5}$. Even in the case of a very strong Li depletion mechanism, such a model cannot account for those CEMPS stars with low ${ }^{12} \mathrm{C} /{ }^{13} \mathrm{C}$ number ratios (see also Sect. 7).

Supernovae imply large dilution factors, therefore such models cannot predict He-rich CRUMPS! Detection of He enhancement in CRUMPS would imply small dilution factors and thus eliminate a strong contribution by supernova ejecta (at least in He-rich CRUMP stars).

\subsection{AGB stars in binary systems}

Many authors argue that the peculiar surface abundances of CEMP stars have been acquired in a mass transfer episode. The most commonly accepted scenario follows the general line that, when the primary has evolved into the AGB phase, part of its wind material is accreted by the secondary (today the CEMP star), giving it the peculiar surface abundances that we can observe today.

The following observed features have been invoked as supporting the AGB binary scenario:

- the presence of $s$-process elements in CEMP-s stars is interpreted as a mark of the AGB nuclesosynthesis;

- the very low iron abundances imply a nucleosynthetic source that does lead to very weak or no iron enrichment. AGB stars do not produce iron;

- binarity indeed seems to be observed (through radial velocity variations) for most of the CEMP-s (Lucatello et al. 2005);

- there seems to be a continuity of properties between the Cenhanced stars and the CH-stars (Masseron et al. 2010);

- a similar argument also seems to hold between the CEMPno and the CEMP- $s$ with a weak overabundance in $s$-process elements (Masseron et al. 2010).

\footnotetext{
5 Looking at Fig. 3 of Tominaga et al. (2007b), it might seem that a star produced from supernova ejecta with high dilution by the ISM would produce Li-depleted stars. This is an artefact because what has been plotted are the ratios of the abundances of these elements with respect to $\mathrm{Fe}$ in the ejecta alone. For heavy elements this is equal to the ratio of the elements after dilution since the primordial matter contains no heavy elements. However, this is not the case for $\mathrm{H}, \mathrm{He}, \mathrm{D}$ and $\mathrm{Li}$. We checked that this interpretation is correct (Tominaga, private communication).
}

In the scenario proposed by Suda et al. (2004) (see also Komiya et al. 2007), the progenitor of the CEMP star was the less massive component in the binary with a mass of the order of $0.8 M_{\odot}$, while the massive component initially had a mass in the range of 1.2-3 $M_{\odot}$. The primary experiences a helium-flash-driven deep mixing as found by Fujimoto et al. (1990). In low-mass models this occurs when the star is at the tip of the red giant branch, in intermediate-mass models, which occurs at the beginning of the AGB phase. As a result, its surface composition changes. During the thermal-pulse AGB phase, additional material is dredged-up to the surface and the primary star emits a wind. Part of the wind is accreted by the secondary and produces the abundance pattern now observed on the surface of CEMP stars. These authors estimate that, in the case of HE 0107-5240 (Christlieb et al. 2004), the amount of mass accreted by the secondary is of the order of about $0.01 M_{\odot}$. (This estimate is obtained by requiring that, when the secondary becomes a red giant, the abundances at its surface match those of HE 0107-5240.) In this scenario the presence of iron-group elements can be attributed either to the binary system forming from a cloud with such an abundance in iron-group elements or by accretion of matter enriched in irongroup elements ejected by one or more first generation supernovae. Since the $s$-process would not be the same in these cases, values of the ratio $[\mathrm{Pb} / \mathrm{Fe}]$ may be used to disentangle these two scenarios (Suda et al. 2004). At the moment current observations do not allow choosing between them.

This scenario presents a reasonable way to explain the CEMP- $s$ stars. Besides explaining the peculiar surface abundances of CEMP stars, it may provide an explanation of why the $\mathrm{C}$-rich stars are more frequent at low metallicity. Let us recall that about $20 \%$ of stars with $[\mathrm{Fe} / \mathrm{H}] \leq-2.0$ exhibit enhancement of the surface carbon abundance (Lucatello et al. 2006), while only $1 \%$ of the Pop II stars are characterized by enhanced $\mathrm{CH}$ bands in the spectra (Luck 1991). The higher frequency of C-rich stars at very low metallicity might be because the heliumflash-driven, deep-mixing phenomenon does appear as a distinct feature of the evolution of extremely metal-poor stars of low and intermediate mass. Also a companion has been detected around 68\% of the CEMP-s stars (Lucatello et al. 2005).

In that scenario the peculiar abundance results from the accretion of a very small amount of material, $0.01 M_{\odot}$ for matching HE 0107-5240, which is a red giant (Suda et al. 2004) ${ }^{6}$ ! Even smaller amounts of material would be needed to reproduce the abundances observed at the surface of unevolved stars presenting similar surface enrichments. This means that such a scenario would obviously not predict He-rich stars.

While this scenario is quite successful in many respects, it also has some drawbacks. For instance, according to Stancliffe \& Glebbeek (2008), (non-rotating) AGB models do not predict substantial enhancements of both carbon and nitrogen at the same time (except in a very narrow mass range). The spinstar AGB model presented previously would provide a way to overcome that difficulty.

\section{Possible origin of CRUMP stars with ${ }^{12} \mathrm{C} /{ }^{13} \mathrm{C}$ below 30}

In this section we want to specifically address the origin of CRUMP stars showing a low ${ }^{12} \mathrm{C} /{ }^{13} \mathrm{C}$ isotopic ratio, typically below 30. In the SAGA databasis, except for two stars, all the

${ }^{6}$ HE 0107-5240 is not a CEMP-s star. Despite that, this star is discussed in detail in the paper by Suda et al. (2004) in the framework of the AGB binary scenario. 
other CEMP-no stars ( 7 stars) present ${ }^{12} \mathrm{C} /{ }^{13} \mathrm{C}$ below 25 . The two exceptions have upper limits in the range of 40-50.

The ${ }^{12} \mathrm{C} /{ }^{13} \mathrm{C}$ isotopic ratio has four properties that make it an excellent probe of various nucleosynthetic environments:

1. in a low-mass main-sequence or subgiant star like HE 1327-2326, there is no known process for modifying this number ratio on the surface. Thus this quantity has the advantage over $\mathrm{Li}$ that its present-day value on the surface of unevolved stars is equal to the initial value of this ratio;

2. the ${ }^{12} \mathrm{C} /{ }^{13} \mathrm{C}$ number ratio presents strong variations depending on the source material being considered;

3. isotopic ratios depend on the nuclear paths, because they are not sensitive to fractionation effects;

4. finally from a nucleosynthetic point of view, low ${ }^{12} \mathrm{C} /{ }^{13} \mathrm{C}$ can only be obtained when a small amount of pure He-burning material is ejected.

The number ratio in the CRUMP star can be expressed as

$\frac{n\left({ }^{12} \mathrm{C}\right)}{n\left({ }^{13} \mathrm{C}\right)}=\frac{13}{12} \frac{X\left({ }^{12} \mathrm{C}\right)_{\text {source }}+X\left({ }^{12} \mathrm{C}\right)_{0} D}{X\left({ }^{13} \mathrm{C}\right)_{\text {source }}+X\left({ }^{13} \mathrm{C}\right)_{0} D}$

where $n$ is the number fraction of the element considered and $X$ its mass fraction in the source material (subscript source) or in the interstellar medium (subscript 0 ). Thus we need to know the values of $X\left({ }^{12} \mathrm{C}\right)_{0}$ and of $X\left({ }^{13} \mathrm{C}\right)_{0}$. Unfortunately, these values are not known for the very low $[\mathrm{Fe} / \mathrm{H}]$ corresponding to CRUMPS. According to Spite et al. (2005), values of $[\mathrm{C} / \mathrm{Fe}]$ around solar values are found in giants with $[\mathrm{Fe} / \mathrm{H}]$ between -4.2 and -2.1 . Spite et al. (2006) give the number ratio ${ }^{12} \mathrm{C} /{ }^{13} \mathrm{C}$ obtained in giants at low $[\mathrm{Fe} / \mathrm{H}]$ (down to about -3.5$)$, which have undergone the first dredge-up but are still below the luminosity bump. Values between 20 and 30 are obtained. These values are lower than the initial value because of the effect of the first dredge-up. According to Chiappini et al. (2008), accounting for this effect implies initial values for ${ }^{12} \mathrm{C} /{ }^{13} \mathrm{C}$ around at least 50 .

Of course there is no guarantee that these values are the same at much lower values of $[\mathrm{Fe} / \mathrm{H}]$. With the chemical evolution model of Chiappini et al. (2008), we obtain values at $[\mathrm{Fe} / \mathrm{H}]=-5$ that differ a lot if considering yields from slow- or fast-rotating models. Slow-rotating models, i.e. with angular momentum lower than for massive stars at solar metallicity, would yield very high values around 31000 ! Fast-rotating models, i.e. with angular momentum content of similar magnitude to that of massive solar metallicity stars, predict ratios equal to 30 . These models are to be preferred since they also reproduce the observed $[\mathrm{N} / \mathrm{O}]$ and $[\mathrm{C} / \mathrm{O}]$ trends with $[\mathrm{Fe} / \mathrm{H}]$ and $[\mathrm{O} / \mathrm{H}]$ in the halo (Chiappini et al. 2006). Regardless of the correct answer, we can at least say that the initial ${ }^{12} \mathrm{C} /{ }^{13} \mathrm{C}$ ratio at very low metallicity is probably greater than 30 .

The effect of dilution is very different depending on the primary or secondary nature of the nucleosynthetic path leading to the synthesis of the two carbon isotopes in the source material. In case both isotopes have a strong primary component, as is the case in spinstar models ${ }^{7}$, then the ${ }^{12} \mathrm{C} /{ }^{13} \mathrm{C}$ ratio will not be affected by dilution much. Typically at $Z=10^{-8}$ the initial abundances of ${ }^{12} \mathrm{C}$ and ${ }^{13} \mathrm{C}$, which we used to compute the models, are $7.55 \times 10^{-10}$ and $0.10 \times 10^{-10}$, respectively, while the abundances in the winds of the $85 M_{\odot}$ model at $Z=10^{-8}$ are 0.097 $\left({ }^{12} \mathrm{C}\right)$ and $0.009\left({ }^{13} \mathrm{C}\right)$ (see Table 1$)$. Thus, for any dilution factors below about $10^{8}$, the number ratio, equal to 11 , is given by $\frac{13}{12} \frac{X\left({ }^{12} \mathrm{C}\right)_{\text {source }}}{X\left({ }^{13} \mathrm{C}\right)_{\text {source }}}$ and is independent of the dilution factor.

${ }^{7}$ Regardless of the spinstar model considered: AGB envelope, wind material, or supernova ejecta.
In the spinstar models, the only way to obtain values for the ${ }^{12} \mathrm{C} /{ }^{13} \mathrm{C}$ ratio below 30 is to consider only wind material of massive stars with no or only a small amount of pure products from He-burning. Models that would lose this envelope, not by winds but at the end of the evolution when they explode as supernova, would eject material with nearly identical compositions to the wind models presented here.

Would rotating AGB models be able to produce low ${ }^{12} \mathrm{C} /{ }^{13} \mathrm{C}$ number ratios? If we consider the mass fractions of the two carbon isotopes in the envelope of our $7 M_{\odot}$ model, we have $8.8 \times 10^{-3}$ for ${ }^{12} \mathrm{C}$ and $0.12 \times 10^{-3}$ for ${ }^{13} \mathrm{C}$. The initial abundances at $Z=10^{-5}$ are $7.55 \times 10^{-7}\left({ }^{12} \mathrm{C}\right)$ and $0.10 \times 10^{-7}\left({ }^{13} \mathrm{C}\right)$ respectively so at least three orders of magnitude lower than the abundances in the envelope at the early AGB phase. If the dilution factor is well below 10000 , then one would obtain a ${ }^{12} \mathrm{C} /{ }^{13} \mathrm{C}$ number ratio equal to 79 , and if the dilution factor is greater than 10000 , one would obtain a ${ }^{12} \mathrm{C} /{ }^{13} \mathrm{C}$ number ratio equal to that of the interstellar medium, between 30 and 31000 (see the discussion above)! Therefore, it does appear that our early-AGB (EAGB) model would not predict very low ${ }^{12} \mathrm{C} /{ }^{13} \mathrm{C}$ number ratio, but this is only one model at a peculiar stage. At the moment, we cannot exclude the possibility that AGB material also yields very low ${ }^{12} \mathrm{C} /{ }^{13} \mathrm{C}$ material.

For non-spinstar models, we discuss here only the case of the supernova models, for which the published data allow easy calculation of the expected ${ }^{12} \mathrm{C} /{ }^{13} \mathrm{C}$ ratio in the CRUMP star. Using the yields of the $40 M_{\odot}$ Pop. III supernovae computed by Tominaga et al. (2007b), we consider their model 40B in their Table 2 . With their mass cut of $10.7 M_{\odot}$, we obtain mass fractions of ${ }^{12} \mathrm{C}$ and ${ }^{13} \mathrm{C}$ in the supernova ejecta equal to 0.012 and $2.7 \times 10^{-9}$. Mixed with primordial material, we obtain a ${ }^{12} \mathrm{C} /{ }^{13} \mathrm{C}$ number ratio equal to $4.4 \times 10^{6}$ independent of the dilution factor. Similar values would be obtained from other models by Tominaga et al. (2007b). This is not a conclusion linked to the peculiar supernova model cited above. The supernova ejecta will be richer than the winds in ${ }^{12} \mathrm{C}$ and, at least, if they are computed from non-rotating models, would be poor in ${ }^{13} \mathrm{C}$. This means that the ${ }^{12} \mathrm{C} /{ }^{13} \mathrm{C}$ number ratio will be very high!

From the above considerations, it appears that an unevolved CEMP star with a ${ }^{12} \mathrm{C} /{ }^{13} \mathrm{C}$ number ratio inferior to about 30 cannot be made from (non-spinstar) Pop III supernova ejecta regardless of the dilution factor. It can be made from the winds of spinstars, even those highly diluted with interstellar material.

\section{Discussion and perspectives}

\subsection{Some general considerations}

In this section, we want to collect some general conclusions that do not depend on a peculiar model for the source of the material responsible for the abundances observed in CEMP stars.

In Table 2, we enumerate 12 observed features (see Col. 2). Each of these is given a number $\mathrm{N}$ (for further reference). It is indicated in Col. 1. Column 4 indicates what can be concluded from the observed feature and the hypothesis given in Col. 3. The CEMP stars are supposed to be composed of some amount of source material, $M_{\text {source }}(\mathrm{Li}$-free) and of interstellar medium material (with WMAP Li). In Col. 3, depletion refers to Li and the symbol "ISM[X/Fe]" indicates the value of the ratio in the interstellar medium at the time of CRUMP/CEMP star formation. In Col. $4, D$ is the dilution factor, "H and He burn" means that the source material from which the CRUMP/CEMP star is made is rich in both $\mathrm{H}$ - and He-burning products, "no He-only" means that this material does not contain (or has very small amounts 
Table 2. Theoretical interpretations of observed features and consequences for helium in CRUMP stars.

\begin{tabular}{|c|c|c|c|c|}
\hline$N$ & Observed feature & Hypothesis & Theoretical interpretation & He-rich \\
\hline 1 & $\epsilon(\mathrm{Li})<$ Spite & no in-situ depl. & $D<\sim 0.5$ & YES \\
\hline 2 & $\epsilon(\mathrm{Li})<$ Spite & strong in-situ depl. & $D>1$ & YES for $D<\sim 4$ \\
\hline 3 & $\epsilon(\mathrm{Li}) \sim$ Spite & & $D>1$, in situ Li- depletion & YES for $D<\sim 4$ \\
\hline 4 & ${ }^{12} \mathrm{C} /{ }^{13} \mathrm{C}<\mathrm{ISM}$ & & H- and He-burn. and no He-only & $?$ \\
\hline 5 & ${ }^{12} \mathrm{C} /{ }^{13} \mathrm{C}>\mathrm{ISM}$ & & $\mathrm{H}$ - and He-burn. plus He-only, $D \gg 1$ & NO \\
\hline 6 & {$[\mathrm{~N} / \mathrm{H}]>1.4+[\mathrm{Fe} / \mathrm{H}]$} & $0 \leq \mathrm{ISM}[\mathrm{CNO} / \mathrm{Fe}] \leq 0.6$ & source material is primary $\mathrm{N}$ rich & $?$ \\
\hline 7 & {$[\mathrm{~N} / \mathrm{C}]$ and $[\mathrm{N} / \mathrm{O}]>0$} & & H- and He-burn. and no He-only & $?$ \\
\hline 8 & {$[\mathrm{~N} / \mathrm{C}]$ and $[\mathrm{N} / \mathrm{O}]<0$} & & H- and He-burn. plus He-only, $D \gg 1$ & NO \\
\hline 9 & {$[\mathrm{Na} / \mathrm{Ne}]$ and $[\mathrm{Al} / \mathrm{Mg}]>0$} & & H- and He-burn. and no He-only, small $D$ & YES \\
\hline 10 & {$[\mathrm{Na} / \mathrm{Ne}]$ and $[\mathrm{Al} / \mathrm{Mg}]<0$} & & H- and He-burn. plus He-only, $D \gg 1$ & NO \\
\hline 11 & {$[\mathrm{Al} / \mathrm{Fe}]>0$} & $\mathrm{ISM}[\mathrm{Mg} / \mathrm{Fe}] \sim 0$ & H- and He-burn. & $?$ \\
\hline 12 & {$[\mathrm{Al} / \mathrm{Fe}]<0$} & & {$[\mathrm{Al} / \mathrm{Fe}]<0$ in $M_{\text {source }}, D \gg 1$} & NO \\
\hline
\end{tabular}

of) matter only processed by He burning, while "+ He-only" means that the source material contains a significant amount of pure He-burning products. Column 5 indicates whether the CRUMP/CEMP star is He-rich (YES) or not (NO), while question mark indicates that both cases are possible. The following general comments are worth making:

1. if $\mathrm{Li}$ is not depleted by the CEMP star itself, then any low observed Li abundance implies a very small dilution factor (feature 1);

2. if $\mathrm{Li}$ is observed to be low and $\mathrm{Li}$ is depleted in the CEMP star, the dilution factor cannot be constrained from the current Li surface abundance (feature 2);

3. if $\mathrm{Li}$ is observed to have a value along the Spite plateau, then the dilution factor cannot be constrained from the current $\mathrm{Li}$ surface abundance and the depletion, if any, would be similar to those in normal halo field stars (feature 3);

4. low ${ }^{12} \mathrm{C} /{ }^{13} \mathrm{C}$ ratios (below 30) indicate that the source material has been strongly processed by the $\mathrm{CNO}$ cycle. The high abundance of carbon (in the form of ${ }^{12} \mathrm{C}$ ) implies a strong primary ${ }^{13} \mathrm{C}$ production. This can occur only if some mixing has occurred in the source between the He- and the $\mathrm{H}$-burning regions (feature 4);

5. high ${ }^{12} \mathrm{C} /{ }^{13} \mathrm{C}$ ratios (above 30 ) indicate either a high dilution factor and/or domination of the source material by Heburning products (feature 5);

6. the source material is rich in primary nitrogen for CRUMP/CEMP stars showing feature 6. A secondary process inside the star can produce at most an $[\mathrm{N} / \mathrm{H}]$ value about 1.4 dex above $[\mathrm{Fe} / \mathrm{H}]$. This is true, of course, as long as the initial abundances of carbon and oxygen are not orders of magnitudes more abundant with respect to $\mathrm{Fe}$ than in the Sun. At the moment, looking at the data by Cayrel et al. (2004), $[\mathrm{C} / \mathrm{Fe}]$ is solar and $[\mathrm{O} / \mathrm{Fe}]$ is about 3 times solar in metal-poor normal halo stars;

7. the $[\mathrm{N} / \mathrm{C}]$ and $[\mathrm{N} / \mathrm{O}]$ ratios are a measure of the importance of the $\mathrm{CNO}$-processed material in the source material. If these ratios are large, this indicates a high proportion of $\mathrm{CNO}$ processed material (feature 7). As indicated many times above,
He-burning products are not absent since they allow the overabundances with respect to iron of carbon and oxygen;

8 . if the $[\mathrm{N} / \mathrm{C}]$ and $[\mathrm{N} / \mathrm{O}]$ ratios are small, this favors Heburning processed material (feature 8 ). This is independent of the dilution factors because all these elements are primary, hence in much greater abundance in the source material than in the interstellar medium. Probably, only an unrealistic large dilution factor can change the ratios obtained in the mixture. One can have $[\mathrm{N} / \mathrm{C}]<0$ and $[\mathrm{N} / \mathrm{O}]>0$ or the inverse in material ejected with both $\mathrm{H}$ - and He-burning material and some pure He-burning material;

9. in a similar way, large $[\mathrm{Na} / \mathrm{Ne}]$ and $[\mathrm{Al} / \mathrm{Mg}]$ ratios point towards material having been processed mainly by the Ne$\mathrm{Na}$ and $\mathrm{Mg}$ - $\mathrm{Al}$ chains active in hot $\mathrm{H}$-burning regions (feature 9). A word of caution, however, has to be given here. Since the pattern of the $\mathrm{Ne}-\mathrm{Na}, \mathrm{Mg}-\mathrm{Al}$ chains manly results from a secondary process, dilution can modify somewhat the final shape of the abundance pattern. Indeed the elements between $\mathrm{Ne}$ and $\mathrm{Al}$ probably have initial abundances within the same order of magnitude, so that the transformations of, for instance, $90 \%$ of $\mathrm{Mg}$ into $\mathrm{Al}$ will produce a contrast between the source material and the interstellar material of at most one order of magnitude. Such a low contrast can thus be erased by a small dilution factor;

10. small $[\mathrm{Na} / \mathrm{Ne}]$ and $[\mathrm{Al} / \mathrm{Mg}]$ ratios are indicative of material processed in He-burning zones (feature 10);

11. feature 11 is compatible with source material processed by the $\mathrm{Mg}-\mathrm{Al}$ chain;

12. feature 12 implies that some iron is ejected by the source;

13. If He-rich CRUMP/CEMP stars exist, they should be found among the stars presenting the features 4, 6, 7, 9, 11 indicated in Table 2. Stars showing these features are however not necessarily He-rich.

\subsection{The main results from our spinstar models}

One of the points from that study is that rotation does play a key role in explaining the abundance pattern observed in CEMP stars. This is true even when the source material comes from 
A\&A 521, A30 (2010)

Table 3. Spinstar models compatible with sets of observed features given in Table 2.

\begin{tabular}{llc}
\hline \hline Observed features & Model & He-rich \\
\hline $1,4,6,7,9,11$ & spinstar wind model & YES \\
$2,4,6,7,9,11$ & spinstar wind model & $?$ \\
$3,4,6,7,9,11$ & spinstar wind model & $?$ \\
$2,5,6,8,10,11$ & spinstar wind and faint supernovae model or spinstar E-AGB model & NO \\
$3,5,6,8,10,11$ & spinstar wind and faint supernovae model or spinstar E-AGB model & NO \\
$2,5,6,8,10,12$ & spinstar wind and faint supernovae model & NO \\
\hline
\end{tabular}

massive star winds, from intermediate mass star winds or from a supernova.

The spinstar scenario that we have presented has consequences for other topical questions related to the chemical evolution of the galactic halo. First, this scenario is indirectly supported by fast-rotating stars seeming needed to reproduce the high N/O plateau observed in the halo of our Galaxy and also the C/O upturn towards low metallicities (Chiappini et al. 2006). An advantage of our scenario is therefore that the same kinds of objects can be responsible for the properties of both normal halo stars and the CRUMPS. The normal ones are made up of a well-mixed reservoir that has been enriched by massive rotating stars of different masses and metallicities as proposed by Hirschi (2007). The CRUMP stars are formed in the vicinity of one massive (or intermediate) rotating star and are made up of material taken from the external layers of such stars.

Second, spinstars may also be invoked to explain the chemically peculiar stars observed in globular clusters ${ }^{8}$. At the metallicity of the globular clusters, fast-rotating stars may lose large amounts of mass when the star reaches the critical limit. This only occurs during the MS phase. The material in the disk is thus only enriched in H-burning products. This material is released very slowly in the cluster's interstellar medium and can thus be retained by its shallow gravitational potential. It can then be used to form stars bearing the signature of H-burning processes as explained in Decressin et al. (2007b,a). When the star evolves further, fast winds develop and escape from the cluster. The same is true for the supernova ejecta.

As sketched in our Fig. 1 we see that some of the CRUMPS stars may receive some SN ejecta (see panel D2). This may explain some r-process enrichment. For the s-process, we think that rotation may strongly affect the nucleosynthesis of these elements and that massive stars might be the source of a much broader range of $s$-process elements than presently believed (Pignatari et al. 2008). Also the impact of rotation in the $s$ process element synthesis in intermediate mass star at very low metallicity also needs to be investigated Siess et al. (2004).

In Table 3, we indicate which spinstar model does the best job of reproducing the various sets of compatible observed features listed in Table 2. Below we draw some conclusions:

1. CRUMP stars originating in the winds of spinstars present strong signs of material processed by $\mathrm{H}$-burning, i.e., high $[\mathrm{N} / \mathrm{C}]$ and $[\mathrm{N} / \mathrm{O}]$ ratios and low ${ }^{12} \mathrm{C} /{ }^{13} \mathrm{C}$ ratios. This is independent of the dilution factor. If the dilution factor is small, these stars are He-rich and $\mathrm{Li}$-poor;

2. the spinstar wind model does appear in the present study as the only model to explain CEMP stars with a low ${ }^{12} \mathrm{C} /{ }^{13} \mathrm{C}$ ratio. It might be that new rotating AGB star models could also

\footnotetext{
8 It is striking that chemically peculiar stars found in the field and in the globular clusters are quite different (rich in both $\mathrm{H}$ - and He-burning products in the field and rich in H-burning products in the clusters). A possible explanation for this fact is proposed in Meynet et al. (2010b).
}

be potential interesting candidates, but this has to be confirmed by further computations;

3. some CRUMPS can also be formed of a mixture of both wind and $\mathrm{SN}$ ejecta from massive fast-rotating stars. Supernovae from non-rotating models cannot reproduce the abundance pattern observed in CRUMPS;

4. stars made of the envelope of E-AGB stars resulting from rotating models also present an abundance pattern in qualitative agreement with CEMP-no stars. Similar non-rotating models do not fit the observed abundances;

5. both the wind+supernova model and the spinstar AGB model require some dilution with a pristine interstellar medium to obtain a reasonable fit. This means that these stars would not be He-rich and would present high ${ }^{12} \mathrm{C} /{ }^{13} \mathrm{C}$ ratios.

\subsection{Why are there no analogs of CRUMPS at higher metallicities?}

One may wonder why the scenario leading to the formation of CRUMPS no longer works in the present universe. Two reasons can be invoked. 1) At high metallicity, rotation cannot produce primary nitrogen, hence the peculiar simultaneous overabundance of CNO elements. 2) At high metallicity, the chemically enriched winds of massive stars propagate in a medium that has already been pushed away by the strong and fast O-type star stellar winds. This may prevent the formation of stars made almost completely of pure chemically enriched ejecta. At very low metallicity, the situation can be very different, the line-driven stellar winds are first very weak, preventing the star from pushing out the circumstellar material. Only when the stellar surface is enriched in heavy elements does the star lose a large amount of material by stellar winds. Therefore, these enriched, slow winds (slow because ejected when the star is a supergiant) will have to pass through a dense circumstellar medium. This may favor the formation of clumps. Further detailed hydrodynamical modelings are needed to confirm or reject these scenarios.

\subsection{How to show that some CRUMPS are He-rich?}

Let us end by saying a few words about the possibility of testing the hypothesis that CRUMPS (or maybe also some CEMP) stars are He-rich. Although detection of He will remain problematic in cool stars, it is not hopeless. Among all the possibilities, let us cite the following ones.

- Helium lines may be observable in cool stars, whose strength might be related to the He abundance. The line He I $10830 \AA$ was used by Moehler et al. (2000) mainly for velocity diagnostics. It is not a straightforward line to interpret because it arises from a very excited metastable level in He I. However, according to Dupree (private communication) if stars of similar $T_{\text {eff }}$ are observed, a relative measure could be done. It might also be possible to get abundances, but that would 
probably need non-LTE modeling ${ }^{9}$. An additional difficulty with this approach comes from the fact that atomic diffusion would deplete the surface in He (see the models by Korn et al. 2009).

- Asteroseismology may help in revealing the He-abundance of these stars.

- We could observe an eclipsing binary system whose components are CRUMPS or CEMP stars and determine the positions of the two components in the HR diagram as well as their masses. The comparison with the stellar models may bring out the need to enhance the He-abundance in order to simultaneously reproduce all the observed features.

- The presence of a significant population of extremely blue horizontal branch stars in the halo field may be an indirect hint of He-rich stars, since such stars can be the descendants of He-rich stars.

\subsection{What to conclude if He-enhancement is detected? What to conclude if He abundance is normal?}

If He-enhancement is detected and found to be a feature that concerns the whole star and not just its surface, then this would definitely imply that the material from which the CRUMPS are made do contain a relatively small amount of interstellar medium. This would discard models explaining CRUMPS as formed by the accretion of a small amount of mass at the top of the secondary in a binary system. It would also discard the normal supernovae as a possible source for the material from which the CRUMPS are made, because the dilution factors used to bring the composition of normal supernova ejecta to the level of the observed abundances in CRUMPS are much too high to make the star He-rich. Faint supernovae with large fallback would remain interesting, as would the effects of rotation for explaining the high nitrogen enhancements. As discussed in the present work, the wind of spinstars would also be a favored candidate in that case. Let us stress here that this model is compatible with the faint supernova model, and both may be linked in a same evolutionary history, rotation allowing enrichment of the envelope in $\mathrm{H}$ - and He-burning products and its ejection as a wind before the faint supernova event. As indicated in the introduction, finding Heenhancement in the CRUMPS will also modify the estimates of the mass of these objects. It may be that the most massive of these He-rich stars now populate the blue end of the horizontal branch.

If He-abundance is found to be normal, i.e. with values near the primordial helium abundance, then it favors either models invoking a small amount of accreted mass (as in the AGB model described above) or models with high dilution factors. In the latter case, the very low abundance of $\mathrm{Li}$ observed in some CEMP stars should be due to depletion mechanism in the star itself. Many of the spinstar models discussed here are compatible with normal He-stars (see Table 3 ). In particular, the wind of spinstars model would remain an interesting model for normal He-stars showing small ${ }^{12} \mathrm{C} /{ }^{13} \mathrm{C}$ number ratios, since combinations of dilution factor and of the initial mass of the spinstar may yield very modest helium enrichments, while still allowing very low ${ }^{12} \mathrm{C} /{ }^{13} \mathrm{C}$ number ratios.

Acknowledgements. The authors thank the referee for constructive and helpful comments and the language editor, J. Adams, for the very careful reading. The authors are very grateful to Dr. Takuma Suda for giving access to the very useful

\footnotetext{
9 In the hotter HB stars $\left(T_{\text {eff }}>10000\right)$ other He lines become visible, and those have been used to determine abundances (see Moehler et al. 2000).
}

SAGA database (http://www.astro.keele.ac.uk/saga/), to Dr. Thomas Masseron for providing the electronic tables of the paper by Masseron et al. (2010) before publication and to Dr. Andrea Dupree for interesting discussions of He lines in cool stars. R. Hirschi acknowledges support from the Marie Curie grant IIF 221145 and an STFC rolling grant.

\section{References}

Andrievsky, S. M., Spite, M., Korotin, S. A., et al. 2008, A\&A, 481, 481

Asplund, M., Grevesse, N., \& Sauval, A. J. 2005, in Cosmic Abundances as Records of Stellar Evolution and Nucleosynthesis, ed. T. G. Barnes III, \& F. N. Bash, ASP Conf. Ser., 336, 25

Bonifacio, P., Molaro, P., Sivarani, T., et al. 2007, A\&A, 462, 851

Bressan, A., Chiosi, C., \& Fagotto, F. 1994, ApJS, 94, 63

Cameron, A. G. W., \& Fowler, W. A. 1971, ApJ, 164, 111

Cayrel, R., Depagne, E., Spite, M., et al. 2004, A\&A, 416, 1117

Chevalier, R. A. 1989, ApJ, 346, 847

Chiappini, C., Hirschi, R., Meynet, G., et al. 2006, A\&A, 449, L27

Chiappini, C., Ekström, S., Meynet, G., et al. 2008, A\&A, 479, L9

Christlieb, N., Gustafsson, B., Korn, A. J., et al. 2004, ApJ, 603, 708

Cyburt, R. H., Fields, B. D., \& Olive, K. A. 2008, J. Cosmol. Astro-Part. Phys., 11,12

Decressin, T., Charbonnel, C., \& Meynet, G. 2007a, A\&A, 475, 859

Decressin, T., Meynet, G., Charbonnel, C., Prantzos, N., \& Ekström, S. 2007b, A\&A, 464, 1029

Decressin, T., Baumgardt, H., Kroupa, P., Meynet, G., \& Charbonnel, C. 2009, IAU Symp. 258, ed. E. E. Mamajek, D. R. Soderblom, \& R. F. G. Wyse, 265

Frebel, A., Collet, R., Eriksson, K., Christlieb, N., \& Aoki, W. 2008, ApJ, 684, 588

Fujimoto, M. Y., Iben, I. J., \& Hollowell, D. 1990, ApJ, 349, 580

Georgy, C., Meynet, G., Walder, R., Folini, D., \& Maeder, A. 2009, A\&A, 502, 611

Han, Z., Podsiadlowski, P., \& Lynas-Gray, A. E. 2007, MNRAS, 380, 1098

Hirschi, R. 2007, A\&A, 461, 571

Joggerst, C. C., Woosley, S. E., \& Heger, A. 2009, ApJ, 693, 1780

Komiya, Y., Suda, T., Minaguchi, H., et al. 2007, ApJ, 658, 367

Korn, A. J., Grundahl, F., Richard, O., et al. 2007, ApJ, 671, 402

Korn, A. J., Richard, O., Mashonkina, L., et al. 2009, ApJ, 698, 410

Limongi, M., Chieffi, A., \& Bonifacio, P. 2003, ApJ, 594, L123

Lind, K., Korn, A. J., Barklem, P. S., \& Grundahl, F. 2008, A\&A, 490, 777

Lucatello, S., Tsangarides, S., Beers, T. C., et al. 2005, ApJ, 625, 825

Lucatello, S., Beers, T. C., Christlieb, N., et al. 2006, ApJ, 652, L37

Luck, R. E. 1991, ApJS, 75, 579

Maeder, A., \& Meynet, G. 2001, A\&A, 373, 555

Maeder, A., Meynet, G., Ekström, S., \& Georgy, C. 2009, Commun. Asteroseismol., 158, 72

Masseron, T., Johnson, J. A., Plez, B., et al. 2010, A\&A, 509, A93

Meneguzzi, M., Audouze, J., \& Reeves, H. 1971, A\&A, 15, 337

Meynet, G., \& Maeder, A. 2002, A\&A, 390, 561

Meynet, G., \& Maeder, A. 2005, A\&A, 429, 581, Paper XI

Meynet, G., Ekström, S., \& Maeder, A. 2006, A\&A, 447, 623

Meynet, G., Decressin, T., \& Charbonnel, C. 2008a, Mem. Soc. Astron. Ital., 79, 584

Meynet, G., Ekström, S., Maeder, A., et al. 2008b, in First Stars III, ed. B. W. O'Shea, \& A. Heger, AIP Conf. Ser., 990, 212

Meynet, G., Ekström, S., Maeder, A., et al. 2008c, ed. F. Bresolin, P. A. Crowther, \& J. Puls, IAU Symp., 250, 147

Meynet, G., Hirschi, R., Ekstrom, S., et al. 2010a, ed. K. Cunha, M. Spite, \& B. Barbuy, IAU Symp., 265, 98

Meynet, G., Hirschi, R., Ekstrom, S., et al. 2010b, [arXiv: 1001.1864]

Moehler, S., Sweigart, A. V., Landsman, W. B., \& Heber, U. 2000, A\&A, 360, 120

Norris, J. E., Christlieb, N., Korn, A. J., et al. 2007, ApJ, 670, 774

O'Connell, R. W. 1999, ARA\&A, 37, 603

Pignatari, M., Gallino, R., Meynet, G., et al. 2008, ApJ, 687, L95

Piotto, G., Bedin, L. R., Anderson, J., et al. 2007, ApJ, 661, L53

Plez, B., \& Cohen, J. G. 2005, A\&A, 434, 1117

Siess, L., Goriely, S., \& Langer, N. 2004, A\&A, 415, 1089

Spite, M., Cayrel, R., Plez, B., et al. 2005, A\&A, 430, 655

Spite, M., Cayrel, R., Hill, V., et al. 2006, A\&A, 455, 291

Stancliffe, R. J., \& Glebbeek, E. 2008, MNRAS, 389, 1828

Suda, T., Aikawa, M., Machida, M. N., Fujimoto, M. Y., \& Iben, I. J. 2004, ApJ, 611,476

Suda, T., Katsuta, Y., Yamada, S., et al. 2008, PASJ, 60, 1159

Tominaga, N., Maeda, K., Umeda, H., et al. 2007a, ApJ, 657, L77

Tominaga, N., Umeda, H., \& Nomoto, K. 2007b, ApJ, 660, 516

Umeda, H., \& Nomoto, K. 2003, Nature, 422, 871

Venn, K. A., \& Lambert, D. L. 2008, ApJ, 677, 572 